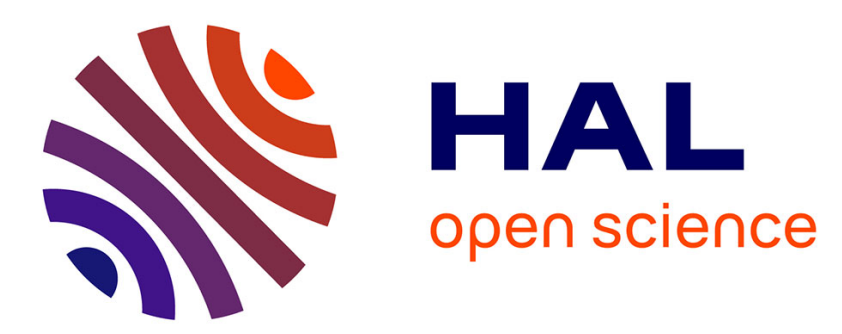

\title{
Time-optimal excitation of maximum quantum coherence: Physical limits and pulse sequences
}

S. Köcher, T. Heydenreich, Y. Zhang, G. N. M. Reddy, S. Caldarelli, H. Yuan, S. J. Glaser

\section{- To cite this version:}

S. Köcher, T. Heydenreich, Y. Zhang, G. N. M. Reddy, S. Caldarelli, et al.. Time-optimal excitation of maximum quantum coherence: Physical limits and pulse sequences. Journal of Chemical Physics, 2016, 144 (16), pp.164103. 10.1063/1.4945781 . hal-01476688

\section{HAL Id: hal-01476688 \\ https://hal.science/hal-01476688}

Submitted on 25 Feb 2017

HAL is a multi-disciplinary open access archive for the deposit and dissemination of scientific research documents, whether they are published or not. The documents may come from teaching and research institutions in France or abroad, or from public or private research centers.
L'archive ouverte pluridisciplinaire HAL, est destinée au dépôt et à la diffusion de documents scientifiques de niveau recherche, publiés ou non, émanant des établissements d'enseignement et de recherche français ou étrangers, des laboratoires publics ou privés. 
S. S. Köcher, ${ }^{1,2}$ T. Heydenreich, ${ }^{1}$ Y. Zhang, ${ }^{1}$ G. N. M. Reddy, ${ }^{3}$ S. Caldarelli, ${ }^{3}$ H. Yuan, ${ }^{4}$ and S. J. Glaser ${ }^{1, a)}$

${ }^{1)}$ Department of Chemistry, Technische Universität München, Lichtenbergstrasse 4, D-85747 Garching,

Germany

2) Institute of Energy and Climate Research (IEK-9), Forschungszentrum Jülich, D-52425 Jülich, Germany

3) Aix Marseille Université, Centrale Marseille, CNRS, iSm2 UMR 7313, 13397, Marseille, France

4) Chinese University of Hong Kong, Department of Mechanical and Automation Engineering, Shatin, N.T. Hong Kong SAR, China

(Dated: 14 March 2016)

Here we study the optimum efficiency of the excitation of maximum quantum (MaxQ) coherence using analytical and numerical methods based on optimal control theory. The theoretical limit of the achievable MaxQ amplitude and the minimum time to achieve this limit are explored for a set of model systems consisting of up to five coupled spins. In addition to arbitrary pulse shapes, two simple pulse sequence families of practical interest are considered in the optimizations. Compared to conventional approaches, substantial gains were found both in terms of the achieved MaxQ amplitude and in pulse sequence durations. For a model system, theoretically predicted gains of a factor of three compared to the conventional pulse sequence were experimentally demonstrated. Motivated by the numerical results, also two novel analytical transfer schemes were found: Compared to conventional approaches based on non-selective pulses and delays, double-quantum coherence in two-spin systems can be created twice as fast using isotropic mixing and hard spin-selective pulses. Also it is proved that in a chain of three weakly coupled spins with the same coupling constants, triple-quantum coherence can be created in a time-optimal fashion using so-called geodesic pulses.

PACS numbers: 02.30.Yy, 03.65.Aa, 43.60.Hj, 76.60.-k, 82.56.-b, 82.56.Jn

Keywords: Multiple quantum excitation; Optimal control; Maximum quantum coherence; Unitary bound; Time-optimal transfer; GRAPE optimization; spectrogram

\section{INTRODUCTION}

The simplification and editing of complicated and overlapping spectra is highly desirable in many applications, such as the analysis of complex mixtures or of large biomolecules. To a certain extent, this can be achieved by using two-dimensional multi-quantum NMR spectroscopy. The largest spectral simplification for homonuclear experiments is obtained by exciting and evolving the maximum quantum (MaxQ) order (also denoted "total spin coherence"1,2) that can be created in a given spin system $^{3-10}$. A variety of pulse sequence elements have been developed for the excitation of multiple quantum coherence ${ }^{3-7}$. The standard pulse sequence element based on non-selective pulses and delays has the basic form $90^{\circ}-\Delta-90^{\circ 4}$. In practice, an additional $180^{\circ}$ pulse is applied in the center of the delay $\Delta$ to refocus chemical shift evolution ${ }^{5}$. The delay $\Delta$ between the $90^{\circ}$ pulses can be optimized based on theoretical transfer functions if all coupling constants are known or it can be determined experimentally to achieve the best signal-to-noise ratio for a given application ${ }^{11}$.

However, in general the excitation of high quantum orders by simple $90^{\circ}-\Delta-90^{\circ}$ sequence elements is quite in-

\footnotetext{
a)Electronic mail: glaser@tum.de
}

efficient. In order to generate MaxQ coherence, this sequence requires that there is at least one spin in the system that is directly coupled to all other spins. Especially for large spin systems, the smallest coupling constant (long-range coupling) forms a bottleneck for the speed of multiple quantum generation, resulting in long interpulse delays $\Delta$ and concomitant signal loss due to relaxation. Significantly improved MaxQ excitation efficiency can be achieved by using more sophisticated sequences adapted to specific coupling topologies ${ }^{1,12}$.

The efficient excitation of multiple quantum coherence is also of interest for multiple quantum filters ${ }^{3,13}$ and for multiple quantum EPR spectroscopy. ${ }^{14-17}$

Ultimately we are interested in the best possible pulse sequence for a given application and a defined set of potential spin systems. More specifically, here we focus on the following questions:

(a) What is the physical limit of the efficiency with which multiple quantum coherence of a desired order can be created in a given spin system with defined coupling constants and resonance frequencies?

(b) What is the shortest possible pulse sequence duration $T^{*}$ that is required to reach this physical limit for a given spin system?

(c) What is the maximum possible efficiency of multiple quantum generation for shorter times, i.e. if the duration $T$ of the pulse sequence is limited to $T<T^{*}$ ? 
answers to questions (a)-(c) would provide benchks tlat allow us to judge the relative performance of Publishilng wn or future pulse sequence. Further questions of immediate practical relevance are:

(d) What is the best possible pulse sequence for a given coupling network with defined coupling constants?

(e) What is the best performance of relatively simple pulse sequences (consisting of a small number of hard pulses and delays) and how large is the achievable gain relative to the simple $90^{\circ}-\Delta-90^{\circ}$ pulse sequence element?

Question (a) can be answered based on the general concept of unitary bounds of spin dynamics ${ }^{18-24}$. Questions (b)-(e) can be explored using optimal-controlbased analytical methods ${ }^{25-28}$ and numerical optimization algorithms, such as GRAPE (gradient ascent pulse engineering) ${ }^{29-33}$ to optimize multiple quantum excitation sequences. Previously, this algorithm has been successfully used for a large range of NMR applications of uncoupled $^{34-37}$ as well as coupled spin systems ${ }^{29,38-42}$, including multiple quantum excitation in solid state $\mathrm{NMR}^{43}$ and the efficient implementation of quantum algorithms ${ }^{44}$. Although it has been demonstrated that relaxation effects can be fully taken into account in GRAPE optimizations ${ }^{29,39,40,45-47}$, for simplicity here we focus on the excitation of MaxQ coherence in liquid state NMR of small molecules, where relaxation effects can be neglected. In this paper we demonstrate general methods of pulse design for excitation of maximum-quantum coherence, focusing on specific exemplary families of spin systems, which are in part motivated by experimental work on mixtures of mono- and polycyclic aromatic hydrocarbons ${ }^{8}$. We discuss the theory and apply it to weakly coupled homonuclear spin systems consisting of up to five spins $1 / 2$. A time-frequency analysis ${ }^{48}$ of the numerically optimized pulse shapes was used to help reveal the principles of their mode of action. The significantly improved performancé of numerically optimized pulse sequences is also demonstrated experimentally.

\section{THEORY}

\section{A. Quality factors for excitation of multiple quantum} coherence

The state of a spin system is characterized by the density operator $\rho(t)$ and its equation of motion is given by the Liouville-von-Neumann equation ${ }^{3}$ :

$$
\dot{\rho}(t)=-i[\mathcal{H}, \rho(t)],
$$

where $\mathcal{H}$ is the total Hamiltonian $\mathcal{H}=\mathcal{H}_{0}+\mathcal{H}_{r f}$ which consist of the free evolution Hamiltonian $\mathcal{H}_{0}$ and the radio-frequency (rf) Hamiltonian $\mathcal{H}_{r f}$.

The free evolution Hamiltonian

$$
\mathcal{H}_{0}=\mathcal{H}_{o f f}+\mathcal{H}_{c}
$$

consists of the offset term

$$
\mathcal{H}_{\text {off }}=\sum_{k=1}^{M} 2 \pi \nu_{k} I_{k z}
$$

where $M$ is the number of spins in the coupling network. In the case of homonuclear spin systems without residual dipolar couplings ${ }^{49}$, the coupling term is isotropic ${ }^{3}$ :

$$
\mathcal{H}_{c}^{i s o}=\sum_{k<l} 2 \pi J_{k l}\left(I_{k x} I_{l x}+I_{k y} I_{l y}+I_{k z} I_{l z}\right) .
$$

In the weak coupling limit $\left(\left|\nu_{k}-\nu_{l}\right| \gg\left|J_{k l}\right|\right)$, the coupling term can be simplified to

$$
\mathcal{H}_{c}^{l o n g}=\sum_{k<l} 2 \pi J_{k l} I_{k z} I_{l z}
$$

during delays or periods of weak rf irradiation. However, note that even in the weak coupling limit the full isotropic coupling term $\mathcal{H}_{c}^{i s o}$ can be recovered by irradiating isotropic-mixing sequences ${ }^{50-52}$. The coupling term in Eq. (5) is often called "weak coupling term". However, here we prefer the term "longitudinal coupling" 53 in order to avoid any confusion that may be created by the fact that in weakly coupled spin systems an isotropic coupling Hamiltonian $\mathcal{H}_{c}^{\text {iso }}$ or a longitudinal effective coupling Hamiltonian $\mathcal{H}_{c}^{\text {long }}$ can be created, depending on the irradiated sequence. For a rigorous definition and a more detailed discussion of the limiting cases considered here, see Appendix A.

The non-selective rf Hamiltonian is given by

$$
\mathcal{H}_{r f}^{\text {non-sel }}=2 \pi\left(u_{x}(t) F_{x}+u_{y}(t) F_{y}\right),
$$

where $u_{x}(t)$ and $u_{y}(t)$ are the amplitudes of the $x$ - and $y$-components of the rf field and $F_{\alpha}=\sum_{k=1}^{M} I_{k \alpha}$ (for $\alpha \in\{x, y, z\})$. In the limit of hard spin-selective pulses ${ }^{38}$ which address each spin individually, the rf Hamiltonian can be expressed as

$$
\mathcal{H}_{r f}^{\text {sel }}=2 \pi \sum_{k=1}^{M}\left(u_{k x}(t) I_{k x}+u_{k y}(t) I_{k y}\right),
$$

see Appendix A for more details.

In the optimizations the goal is to find pulse sequences that steer a given initial density operator $\rho(0)$ in a specified time $T$ to a density operator $\rho(T)$ which has maximum overlap with a desired target operator. Starting at thermal equilibrium, the initial density operator is proportional to

$$
\rho(0)=F_{z}=\sum_{k} I_{k z}
$$

and the target state of the density operator is the multiple quantum operator

$$
P^{+}=\prod_{k=1}^{M} I_{k}^{+}=\prod_{k=1}^{M}\left(I_{k x}+i I_{k y}\right)
$$


This manuscript was accepted by J. Chem. Phys. Click here to see the version of record.

$$
Q=\frac{\left|\left\langle\rho(T) \mid P^{+}\right\rangle\right|}{\|\rho(0)\| \cdot\left\|P^{+}\right\|}
$$

i.e. by the absolute value of the scalar product between the (normalized) final density operator $\rho(T)$ and the (normalized) target operator $P^{+}$, where $\|\rho(T)\|=$ $\|\rho(0)\|$ if relaxation can be neglected. A given initial pulse can be efficiently optimized in an iterative fashion using the GRAPE algorithm in combination with a finite difference gradient ${ }^{29,31,32}$.

\section{B. Approaches of pulse sequence optimization}

Here we consider three different approaches of pulse sequence optimization, corresponding to three major classes of pulse sequences (c.f. Fig. 1, for more details see Appendix A and section I of the Supplementary Material $^{55}$ ):

Approach I: In the most general approach, arbitrary pulse shapes $u_{x}(t)$ and $u_{y}(t)$ are considered that are irradiated during a given duration $T$ (c.f. Fig. 1 A). We consider weakly coupled homonuclear spin systems, where the spins are assumed to have large offset differences compared to $J_{\max }$, where $J_{\max }$ is the largest coupling constant in the spin system as discussed in section II A and in Appendix A.

A detailed analysis showed that it is helpful to consider four limiting cases: isotropic (iso) and longitudinal (long) coupling both with non-selective (non-sel) or spin-selective (sel) pulses (c.f. Table II, Fig. 6 and Appendix A). This distinction is important to qualitatively and quantitatively understand the theoretically achievable limits and to put the numerically obtained optimization results in perspective. Even in the presence of large offset differences it is possible to recover the full isotropic coupling term $\mathcal{H}_{c}^{\text {iso }}$, e.g. in isotropic mixing experiments $^{50,52}$. However, numerical tests showed that the optimization algorithm is likely to be trapped in local maxima for relatively weak initial pulse amplitudes and hence is often not able to reach the case of isotropic mixing (data not shown), In addition, as discussed below, some transfer schemes are based on spin-selective rotations. Although such selective rotations can always be realized using a non-selective rf Hamiltonian $\mathcal{H}_{r f}^{n o n-s e l}$ (c.f. Eq. (6)) and delays (assuming the spins have different offset frequencies due to different chemical shifts), the resulting pulse sequence durations strongly depend on the offset differences in the spin system. In these cases, a much simpler, offset-independent picture results if one considers the limiting case of hard selective pulses with negligible duration using the rf Hamiltonian $\mathcal{H}_{r f}^{\text {sel }}$ (c.f. Eq. (7) $)^{38}$.

Approach II: In the second approach (c.f. Fig. 1 B), we considered sequences of $N_{B}$ basic building blocks ${ }^{54}$ where

A

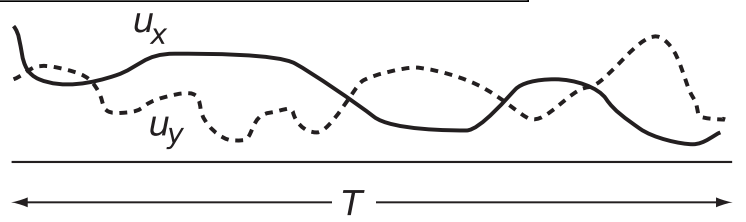

B

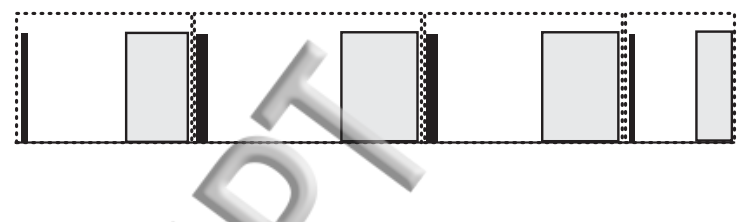

C

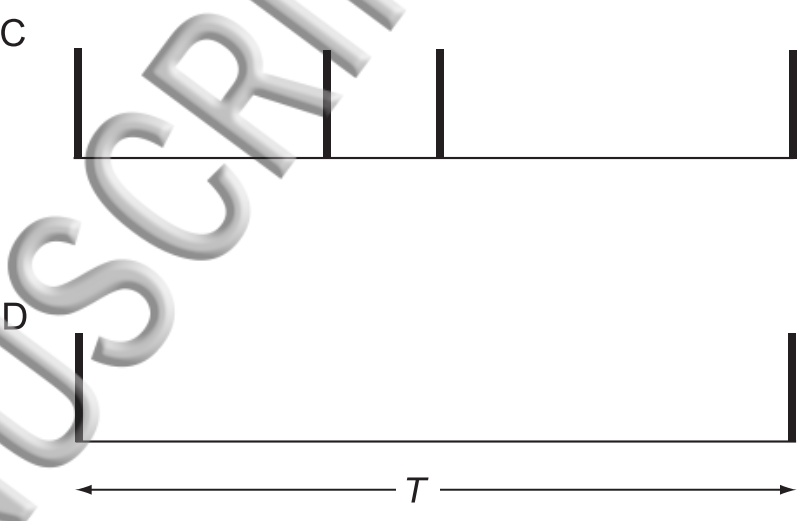

FIG. 1. Schematic representation of the families of pulse sequences considered here for excitation of multiple quantum coherence: (A) arbitrary pulse shapes $u_{x}(t)$ and $u_{y}(t)$ of duration $T$ that are optimized in approach $I$, (B) sequence of $N_{B}$ basic building blocks (represented by dotted boxes) that is used in approach II, where in the given example the number of blocks $N_{B}$ is four. Each block consists of a hard pulse of arbitrary flip angle $\alpha_{k}$ and phase $\varphi_{k}$, a period $\Delta_{k}^{\text {long }}$ of longitudinal coupling evolution and an optional period $\Delta_{k}^{i s o}$ of isotropic mixing (represented by a grey box; approach II (iso/long)), (C) sequences consisting of $N_{P} 90^{\circ}$ pulses separated by periods $\Delta_{k}^{\text {long }}$ of longitudinal coupling evolution, where in the given example $N_{P}=4$. The $\left(N_{P}-1\right)$ delays $\Delta_{k}^{\text {long }}$ are optimized in approach III. The phases $\varphi_{k}$ of the pulses are fixed to either $x$ or $y$ and combinations of these phases were considered, (D) basic $90^{\circ}-\Delta-90^{\circ}$ sequence with phase difference $\varphi_{2}-\varphi_{1}=\pi / 2$ or 0 for excitation of multiple quantum coherence of odd or even order, respectively ${ }^{4,5}$.

each block consists of (a) a hard pulse of arbitrary flip angle $\alpha_{k}$ and phase $\varphi_{k}$, (b) a period $\Delta_{k}^{\text {long }}$ of longitudinal coupling evolution and (c) an optional period $\Delta_{k}^{i s o}$ of isotropic mixing (approach II (iso/long)). In practice, we focused on sequences consisting of $N_{B}=4$ and $N_{B}=16$ blocks and the sequence parameters $\alpha_{k}, \varphi_{k}, \Delta_{k}^{\text {long }}$ and $\Delta_{k}^{i s o}$ were optimized for $1 \leq k \leq N_{B}$ (c.f. section II B of the Supplementary Material ${ }^{55}$ ).

Approach III: In the third approach we focused on simple sequences consisting only of $N_{P}$ pulses with fixed flip angles of $90^{\circ}$ separated by periods $\Delta_{k}^{\text {long }}$ of longitudinal 
$A \mid P_{0-6}$

Publishing

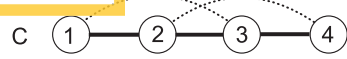

D

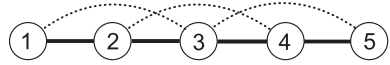

$E(1) \frac{J_{\text {max }}}{\frac{J_{\text {max }}}{2}}(3$

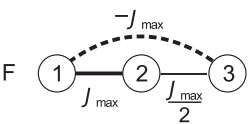

FIG. 2. Schematic representation of the considered idealized coupling topologies: (A-D) show spin chains consisting of up to five spins $1 / 2$. The corresponding offsets are given in Table I. In addition to the the case of only nearestneighbor couplings $J_{k, k+1}=J_{\max }$ (solid lines), we also consider the case of additional second-nearest neighbor couplings $J_{k, k+2}=J_{\max } / 7$ (dottet lines). (E) shows the case of a threespin chain with unequal coupling constants $\left(J_{12}=J_{\max }\right.$, $\left.J_{23}=J_{\max } / 2\right)$ and $(\mathrm{F})$ shows the case of a triangular coupling topology with couplings $J_{12}=J_{\max }, J_{13}=J_{\max } / 2$ and $J_{23}=-J_{\max }$.

TABLE I. Summary of the assumed relative offsets $\nu_{k} / J_{\max }$ and coupling constants $J_{k, l} / J_{\max }$ for spin systems A-F (c.f. Fig. 2), where the asterisk $\left(^{*}\right)$ denotes second-nearest neighbor coupling constants that by default are assumed to be zero, except in Figs. 4 and 5 where also second-nearest neighbor coupling constants $J_{k, k+2}=J_{\max } / 7$ (c.f. dotted lines in Fig. 2) are considered for approach III.

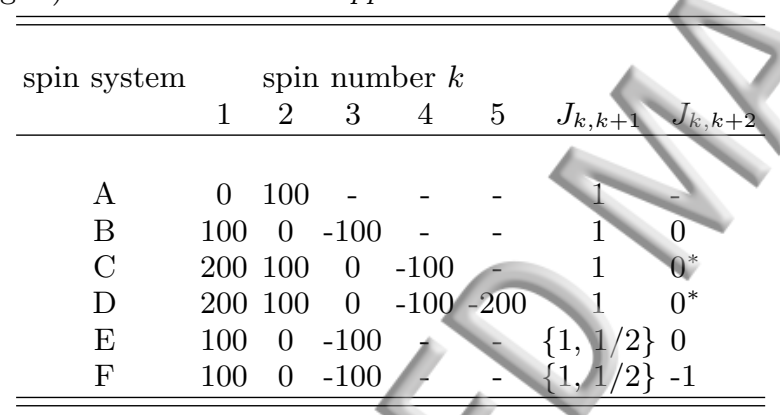

coupling evolution (c.f. Fig. 1 C). For systems consisting of up to four spins, the phases $\varphi_{k}$ of the pulses were fixed to either $x$ or $y$ and all possible combinations of these phases were investigated. For five spins, only the case of identical pulse phases was considered.

The pulse sequences resulting from approaches $I I$ and III can be made broadband by inserting $180^{\circ}$ refocusing pulses in the delays (approaches $I I$ and $I I I$ ) and by using broadband isotropic mixing sequences (approach II).

\section{RESULTS AND DISCUSSION}

\section{A. Idealized spin systems describing molecular coupling topologies}

We investigated the efficiency of MaxQ excitation for the idealized spin systems shown in Fig. 2. Motivated by
A
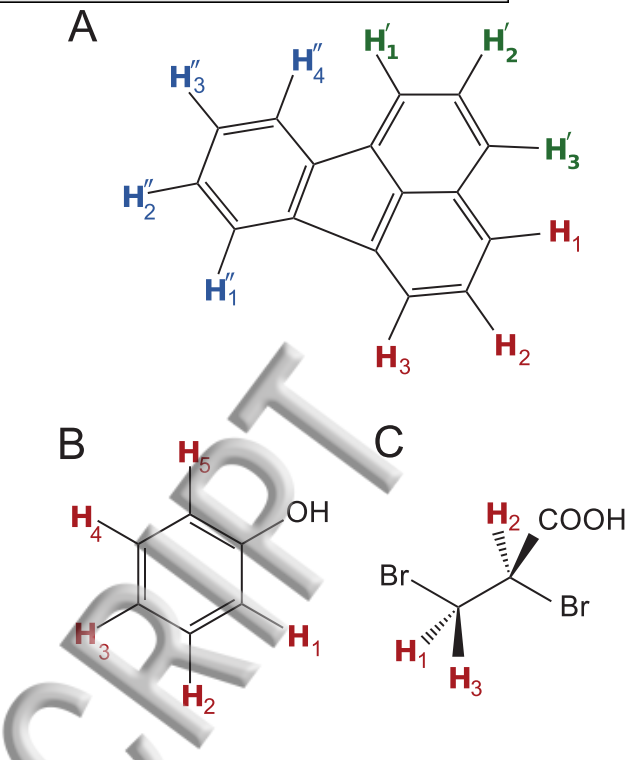

FIG. 3. Examples of ${ }^{1} \mathrm{H}$ spin systems that are approximately represented by some of the idealized coupling topologies shown in Fig. 2: (A) In fluoranthene, the protons labeled $\mathrm{H}_{1}-\mathrm{H}_{3}$ (red) and $\mathrm{H}_{1}^{\prime}-\mathrm{H}_{3}^{\prime}$ (green) form two (approximately isolated) three-spin chains (Fig. $2 \mathrm{~B}$ ) and the protons labeled $\mathrm{H}_{1}^{\prime \prime}-\mathrm{H}_{4}^{\prime \prime}$ (blue) form a four-spin chain (Fig. $2 \mathrm{C}$ ). (B) The proton spin system of phenol can be approximated by a fivespin chain (Fig. 2 D). (C) The proton spin system of 2,3dibromopropionic acid with $J_{12}=11.4 \mathrm{~Hz}, J_{23}=4.4 \mathrm{~Hz}$, and $J_{13}=-10.15 \mathrm{~Hz}$ is approximated by the idealized triangular coupling topology of Fig. 2 F.

previous MaxQ experiments on mixtures of mono- and polycyclic aromatic hydrocarbons ${ }^{8}$, we studied idealized linear chains of coupled spins with only nearest-neighbor couplings $J_{k, k+1}=J_{\max }$ (solid lines in Fig. 2). In addition, we also considered the more realistic case of additional second-nearest neighbor couplings $J_{k, k+2}$ (dotted lines in Fig. 2). Fig. 3 shows examples of molecules with coupling topologies that are approximated by some of the idealized coupling topologies show in Fig. 2. For example, for the ${ }^{1} \mathrm{H}$ spin systems of mono- and polycyclic aromatic hydrocarbons such as fluoranthene and phenol (c.f. Fig. $3 \mathrm{~A}$ and B), typical $J_{k, k+2} / J_{\max }$ ratios are in the order of $1 / 7$. Long range $J_{k, k+3}$ couplings, which are typically at least one order of magnitude smaller, are neglected here. The relative size of the coupling constants of the idealized spin system shown in Fig. $2 \mathrm{~F}$ closely approximate the situation in the ${ }^{1} \mathrm{H}$ spin system of 2,3-dibromopropionic acid (c.f. Fig. 3 C) with the experimentally determined coupling constants $J_{12}=11.4$ $\mathrm{Hz}, J_{23}=4.4 \mathrm{~Hz}$, and $J_{13}=-10.15 \mathrm{~Hz}^{56}$. The coupling network shown in Fig. $2 \mathrm{E}$ forms an intermediate case between Fig. $2 \mathrm{~B}$ and $2 \mathrm{~F}$.

Based on numerical optimizations for each coupling topology, we systematically optimized the efficiency of MaxQ excitation as a function of the pulse sequence duration $T$ using approaches I-III. The results are summarized in Figs. 4, 5 and 6. The unitary bound $Q_{\max }{ }^{18-24}$ 
1 for the achievable efficiency of MaxQ excitation (c.f. TaII) is indicated in each panel of Figs. 4 and 5 by a Publisdiviogtal dotted line.

\section{B. Approach I: TOP curves and unitary bounds}

The maximum possible MaxQ excitation efficiency as a function of pulse sequence duration can be explored numerically using approach $I$ and its graphical representations (marked by solid circles in Figs. 4 and 5 for $a p$ proach I (iso; sel)) are called TOP (time optimal pulse) curves $^{25,38}$. The numerical TOP curves provide a benchmark to judge the relative performance of conventional pulse sequences and of simple sequences based on approach II or III. The shaded areas in Figs. 4 and 5 represent the "forbidden" regions of the graphs, i.e. all possible experimental MaxQ excitation schemes are bounded by the TOP curve. For increasing pulse durations, the TOP curves closely approach the theoretical unitary bounds.

In order to determine the best numerical estimate of the minimum times $T^{*}($ num $)$ and $T_{\text {long }}^{*}($ num $)$ for the case of isotropic and longitudinal coupling, respectively, the logarithm of the difference between the unitary bound $Q_{\max }$ and the numerically obtained optimal quality factors $Q(T)$ for approach I (iso; non-sel) (black open diamonds), approach I (iso; sel) (red circles) and approach I (long; sel) (blue squares) is plotted in Fig. 6. Note that the limiting cases of approach I (iso; non-sel) with $\left|\nu_{k}-\nu_{l}\right| \gg J_{\max }$ and approach I (iso; sel) with hard spinselective pulses should result in identical TOP curves. However as discussed in section IIB, for the case of finite offset differences $\left|\nu_{k}-\nu_{l}\right|$, it is expected, that the optimizations with approach I (iso; non-sel) yield longer pulse durations in comparison to approach I (iso; sel). For cases B to F, the deviations are only on the order of $10 \%$ (c.f. Fig. 6). For case A, the numerical optimization of non-selective pulses starting from random sequences surprisingly yielded durations about twice as long as the pulse durations found for hard spin-selective optimizations. This is due to trapping in local maxima because $T^{*}$ is closely approached when starting with the analytical solution discussed in section IIIF. The minimum times $T^{*}($ num $)$ and $T_{\text {long }}^{*}($ num $)$ found to approach the unitary bound with an error of less than $10^{-5}$ in the isotropic and longitudinal coupling limit are defined in Fig. 6 as the intersections of the horizontal dashed line $\left(\log \left(Q_{\max }-Q\right)=-5\right)$ with the red solid curve and the blue solid curve, respectively. The numerically determined values $T^{*}($ num $)$ and $T_{\text {long }}^{*}($ num $)$ are summarized in Table II. As expected for the case of linear spin chains $T^{*}$ (num) increases monotonically with increasing chain length from $0.26 / J_{\max }$ for $M=2$ to $0.90 / J_{\max }$ for $M=5$ due to the necessity of additional relay steps.
C. Conventional approach $90^{\circ}-\Delta-90^{\circ}$ (approach III with $N_{p}=2$ pulses)

In addition to the unitary bound and the TOP curves, in each panel of Fig. 4 the MaxQ excitation efficiency of the basic $90_{y}^{\circ}-\Delta-90_{\varphi}^{\circ}$ pulse sequence element (with $\varphi=x$ if $M$ is odd and $\varphi=y$ if $M$ is even ${ }^{5}$ ) is shown by red solid curves for comparison (assuming $J_{k, k+2}=0$ for the spin chains in Fig. 2 B-E).

The basic two-pulse sequence reaches the unitary bound only for the two-spin system (Fig. 4 A), but a pulse duration of $T=0.5 / J_{\max }$ is necessary, which is about twice as long as the minimum time $T^{*}$ (num) of approach I (iso; sel). For the considered three-spin systems, the basic two-pulse sequence reaches only about $33 \%$ of the unitary bound (Figs. 4 B, E, F). No MaxQ coherence can be created by the simple two-pulse sequence for spin chains consisting of four and five spins (Fig. $4 \mathrm{C}$ and D) if only nearest-neighbor couplings are non-zero $\left(J_{k, k+2}=0\right)$. This is expected as in this case there exists no spin that is coupled to all remaining spins in the coupling network (see Fig. 2 C, D as well as section III B of the Supplementary Material ${ }^{55}$ ). Only for the case of non-vanishing second-nearest neighbor coupling $\left(J_{k, k+2} \neq 0\right)$, MaxQ coherence is created by the simple two-pulse sequence, see dashed red curves (--) in Fig. $4 \mathrm{C}$ and D. Even in this case, the transfer efficiency of the two-pulse sequence is small compared to the unitary bound $(50 \%$ at $T=3.5 J_{\max }^{-1}$ for 4 spins; $20 \%$ at $T=3.5 J_{\max }^{-1}$ for 5 spins) and the buildup of MaxQ coherence is slow. The low transfer efficiency is explained by the fact that only a fraction of the spins are coupled to all other spins of the chain. For example, in the five-spin chain (Fig. 4 D), only the center spin $\left(I_{3}\right)$ is coupled to all remaining spins of the chain. Hence, only the polarization $I_{3 z}$ can serve as a source of MaxQ coherence, whereas the polarizations $I_{1 z}, I_{2 z}, I_{4 z}$ and $I_{5 z}$ cannot be used by this simple pulse scheme. The slow buildup of MaxQ coherence is a result of the bottleneck formed by the relatively small second-nearest neighbor couplings, chosen here as $J_{13}=J_{35} \approx J_{\max } / 7$ as for the basic two-pulse experiment the transfer function is proportional to the product $\sin \left(\pi J_{13} T\right) \sin \left(\pi J_{23} T\right) \sin \left(\pi J_{34} T\right) \sin \left(\pi J_{35} T\right)$ in the fivespin chain.

\section{More than two $90^{\circ}$ pulses (approach III with $N_{p}>2$ pulses)}

Significantly larger and faster excitation of MaxQ coherence can be achieved using more than two $90^{\circ}$ pulses (approach III). In Fig. 5, the green solid curves represent the achievable transfer efficiency based on approach III for the case of $N_{p}=M$, where $M$ is the number of spins, assuming $J_{k, k+2}=0$ for the spin chains in Fig. 2 A-D.

All possible combinations of pulse phases $x$ or $y$ were simulated for systems consisting of up to four spins and only the curves for the best combinations are presented 


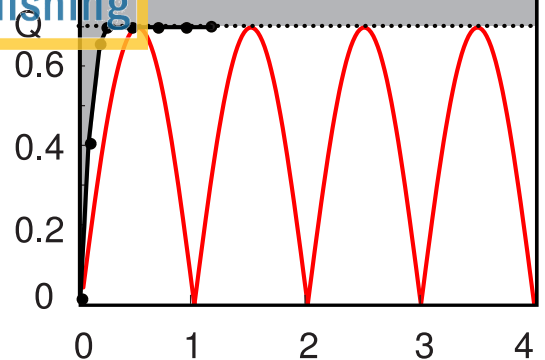

This manuscript was accepted by J. Chem. Phys. Click here to see the version of record.
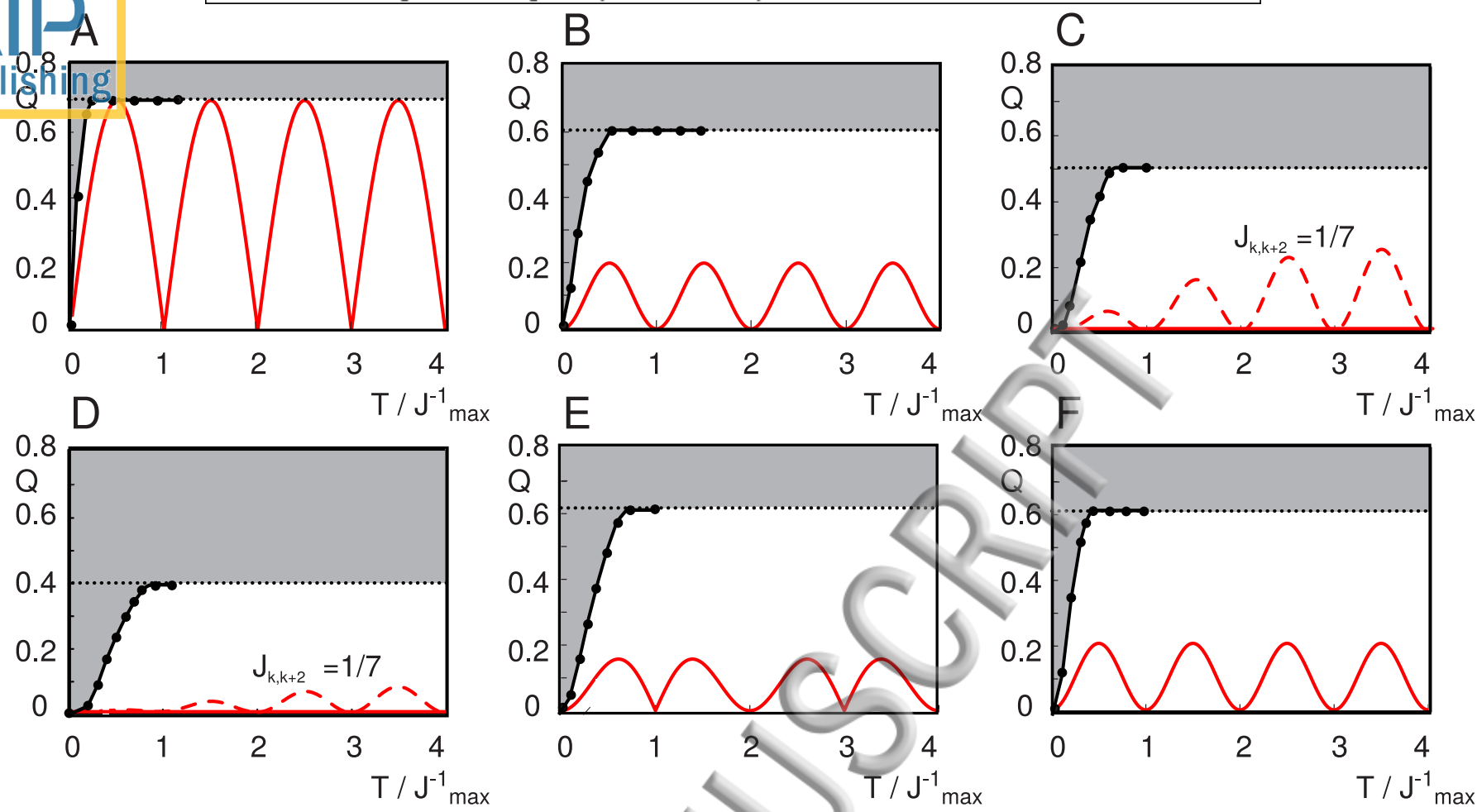

FIG. 4. Panels A-F show the maximum quantum (MaxQ) excitation efficiency $Q$ (c.f. Eq. (10)) for several excitation schemes as a function of pulse sequence duration $T$ for the corresponding idealized coupling topologies of Fig. 2 A-F. The unitary bound for the maximum efficiency of MaxQ generation (c.f. Table II) is indicated in each panel by a horizontal dotted line, the maximum achievable efficiency $Q$ for any given pulse sequence duration $T$ is given by the time-optimal pulse (TOP) curve (marked by solid circles), which is found using approach $I$ (iso; sel) for the case of isotropic coupling with $J_{k, k+2}=0$. The shaded area limited by the TOP curve and the unitary bound represents the "forbidden" regions of the graphs. The achievable transfer efficiency of the conventional building block consisting of two $90^{\circ}$ pulses (corresponding to approach III with $N_{p}=2$ pulses) is represented by red curves. For the case $J_{k, k+2}=J_{\max } / 7$ (c.f. panels C, D, asterisk in Table I), the corresponding transfer functions are represented by dashed curves (--) of the same color. Since the three-spin ring system F is already defined by a non-zero second-nearest neighbor coupling $J_{13}=-J_{\max }$, the transfer function of the conventional sequence is depicted by a solid curve. The pulse sequence duration $T$ is given relative to the inverse of the largest coupling constant $J_{\max }$ in each spin system.

TABLE II. For the spin systems represented in Fig. 2 A-F, the table summarizes the unitary bound for the maximum quantum excitation efficiency $Q_{\text {max }}$. $M$ is the number of spins in the coupling network. $T^{*}$ denotes the minimum pulse duration necessary to approach the unitary bound in the isotropic mixing regime (c.f. Eq. (4)), whereas $T_{\text {long }}^{*}$ denotes the results in the longitudinal coupling limit (c.f. Eq. (5)). The fourth and sixth columns give the numerically found estimates $T^{*}(n u m)$ and $T_{\text {long }}^{*}($ num $) . T^{*}($ num $)$ and $T_{\text {long }}^{*}($ num $)$ are calculated based on the optimizations with approach $I$ (iso; sel) and approach $I$ (long; sel), respectively (c.f. Fig. 6). The analytical minimal pulse duration $T^{*}($ ana $)$ for the two-spin system A as well as $T_{\text {long }}^{*}($ ana $)$ for the two-spin system A and the three-spin system B are derived in sections IV and V of the Supplementary Material $^{55}$ and summarized in the fifth and seventh column.

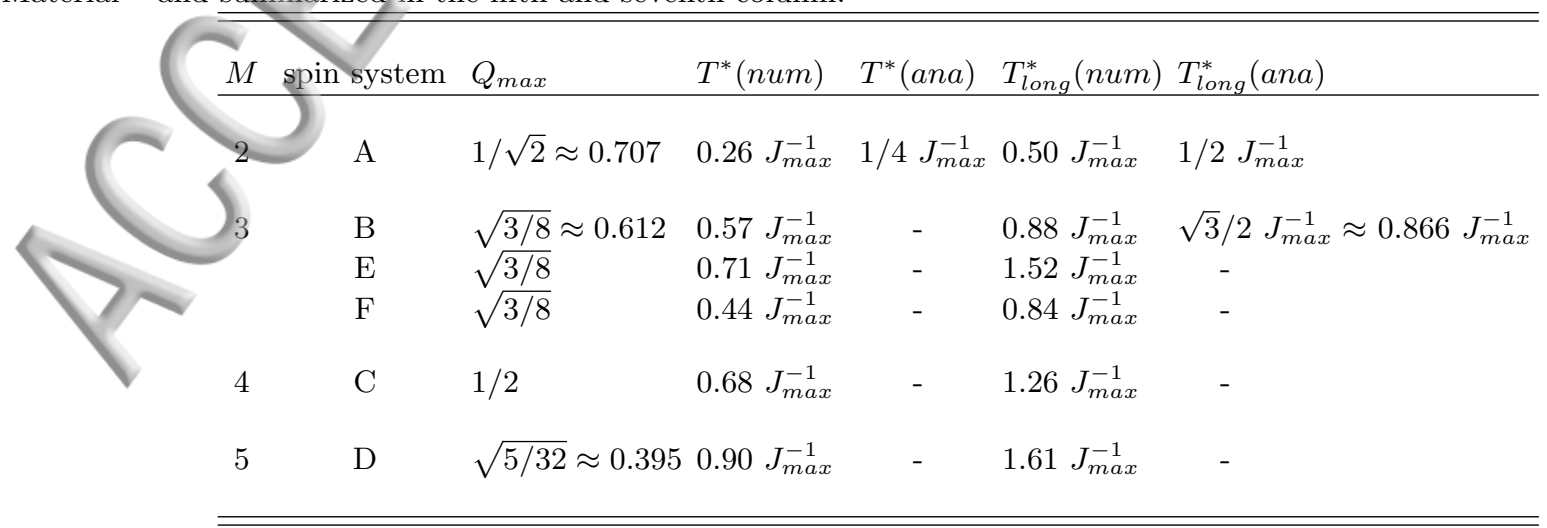




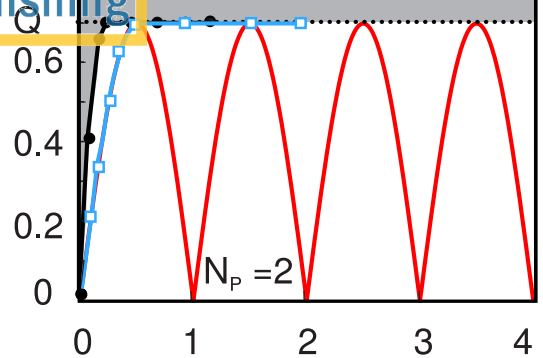

This manuscript was accepted by J. Chem. Phys. Click here to see the version of record.
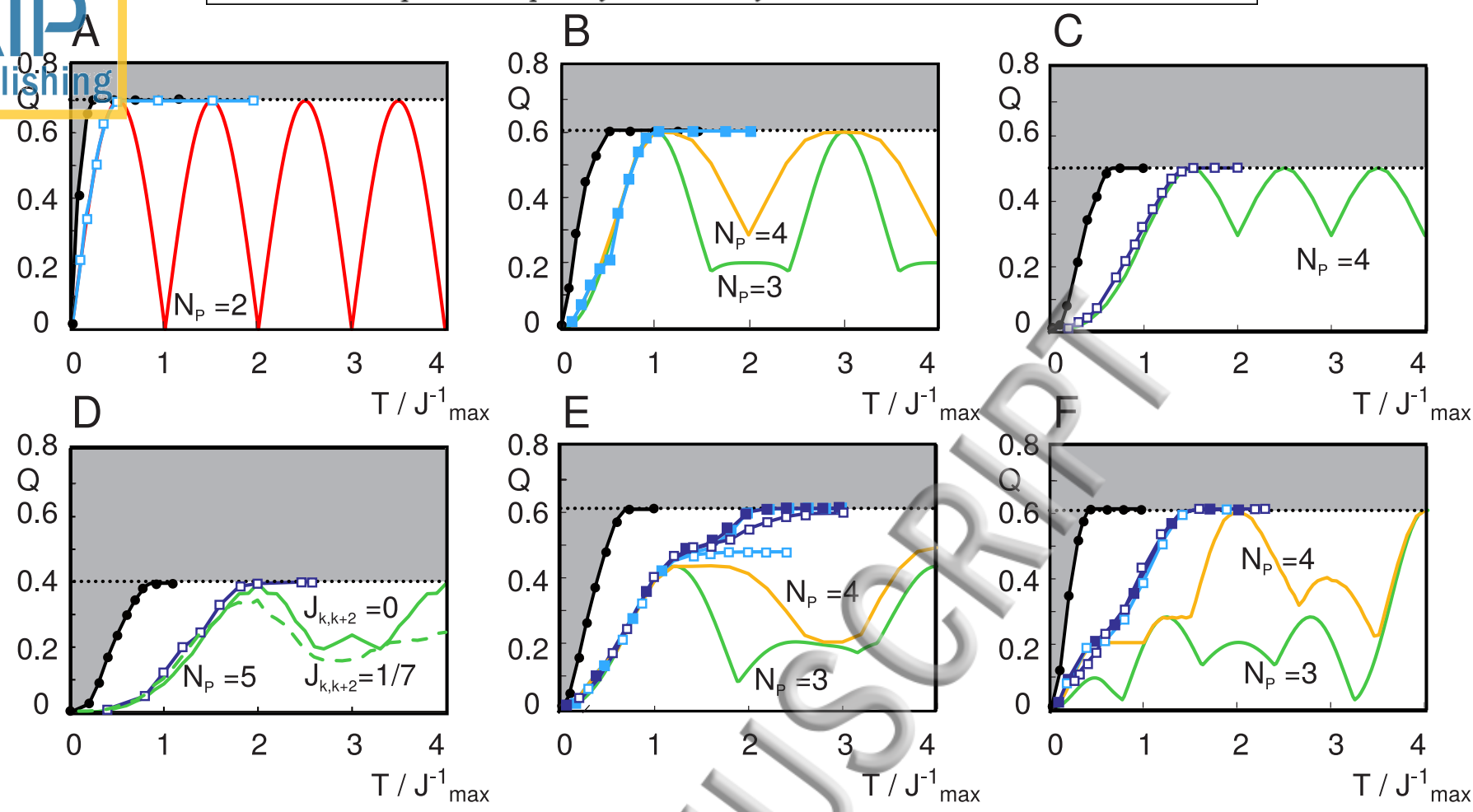

FIG. 5. In addition to the TOP curves (solid black curve with solid circles) for the coupling topologies A-F, the transfer efficiencies achievable by pulses optimized using approach $H I$ with $N_{p}=M$ pulses and $M+1$ pulses (where $M$ is the number of spins) are represented by green and orange curves, respectively, assuming $J_{k, k+2}=0$. Note that for system A with $M=2$, the red curve from Fig. 4 A corresponds to $N_{p}=M$. For the case $J_{k, k+2}=J_{\max } / 7$ (c.f. asterisk in Table I), the corresponding transfer functions are represented by dashed curves $(-\rightarrow)$ of the same color (c.f. panel D). Since the three-spin ring system F is already defined by a non-zero second-nearest neighbor coupling $J_{13}=-J_{\max }$, the transfer efficiency achievable by approach $I I I$ is depicted by a solid curve (green and orange for $N_{p}=M$ pulses and $M+1$ pulses, respectively). Open and solid squares

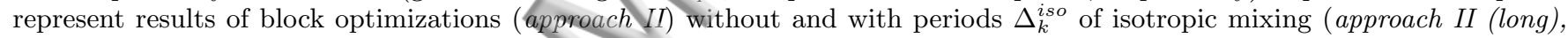
approach II (iso/long)), respectively. Squares connected by light blue lines correspond to the optimization of $N_{B}=4$ blocks, whereas squares connected by dark blue lines correspond to the optimization of $N_{B}=16$ blocks.

in Fig. 5 (see section II C and Table S2 in the Supplementary Material ${ }^{55}$ ). The studies of the five spin system (Fig. 5 D) were limited to the case where all pulse phases are identical. For the spin chains (Fig. 2 A-D), approach III yields simple and quite efficient pulse sequences for $N_{p}=M$ pulses. The necessary time to reach the unitary bound is $1.92 T^{*}$ for $M=2$ spins, $1.75 T^{*}($ num $)$ for $M=3$ spins, $2.21 T^{*}$ (num) for $M=4$ and $2.22 T^{*}($ num $)$ for $M=5$ spins. The resulting pulse sequences can be analyzed in a straightforward way using the product operator formalism (see sections III.A and III B of the Supplementary Material ${ }^{55}$ for two illustrative examples). For the case of a linear three-spin chain with equal coupling constants $J$ (spin system B), the optimal sequence consists of three $90^{\circ}$ pulses with identical phases separated by delays of $1 /(2 J)$, which is identical to the sequence proposed in references 1 and 12 for an $\mathrm{AX}_{2}$ system corresponding to a linear three-spin topology.

While for the case of linear chains (or symmetric coupling topologies) with equal coupling constants it is possible to derive sequences with reasonable transfer efficiencies by hand using the well-known rules of the product operator formalism ${ }^{1,12}$, finding optimal pulse sequences in the case of unequal couplings or complex coupling networks is a highly non-trivial task. The effect of including second-nearest neighbor couplings is shown in Fig. $5 \mathrm{D}$ for $N_{p}=M=5$. In contrast to the case of the two-pulse sequence (Fig. $4 \mathrm{D}$ ), the dashed green line (for the case $J_{k, k+2}=J_{\max } / 7$ ) and the solid green line (for the case $\left.J_{k, k+2}=0\right)$ are quite similar because the sequences exploit the large nearest-neighbor couplings. Only for short pulse durations $T<1.6 / J_{\max }$, the achieved MaxQ coherence of the five-pulse sequence can be slightly improved by taking the $J_{k, k+2}$ couplings into account. Similar results are found for the three and four spin chains (data not shown).

For the three-spin system of Fig. $2 \mathrm{E}$ with unequal couplings, the largest transfer amplitude of the sequence consisting of $N_{p}=M=3$ pulses $\left(90^{\circ}\right)$ is only about $71 \%$ of the unitary bound (c.f. Fig. 5 E). For the threespin systems of Fig. $2 \mathrm{~F}$, the unitary bound is reached, but only for a relatively long pulse duration of $4 J_{\max }^{-1} \approx$ $9.09 T^{*}($ num) (c.f. Fig. $5 \mathrm{~F}$ ). Increasing the number of $90^{\circ}$ pulses from $N_{p}=M=3$ to $N_{p}=M+1=4$ (or- 

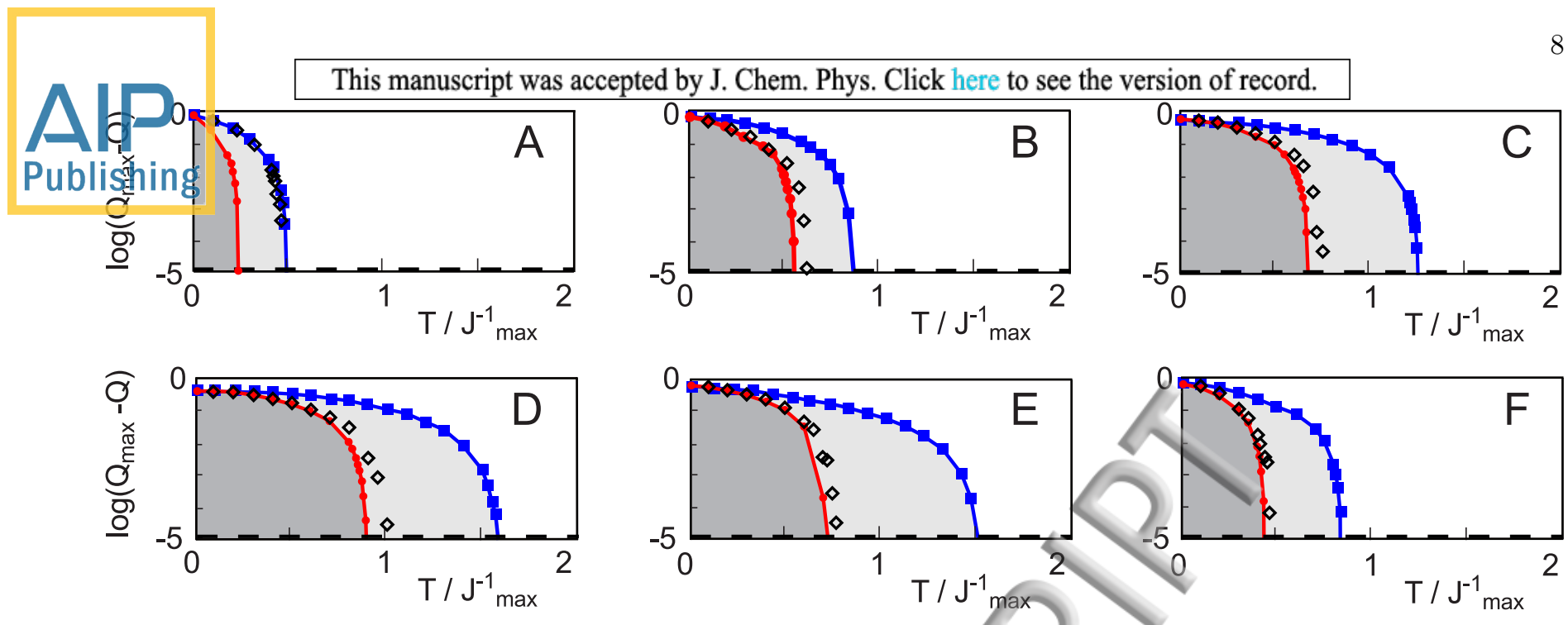

FIG. 6. The logarithm of the difference between the unitary bound $Q_{\max }$ and the numerically obtained optimal quality factors $Q(T)$ for the pulse sequences optimized with approach $I$ as a function of the pulse sequence duration $T$ is shown. The red circles (connected by red lines to guide the eye) correspond to the idealized limiting case of hard spin-selective pulses in the presence of isotropic couplings, approach I (iso; sel). Similarly, the blue squares (connected by blue lines) depict the idealized limiting case of hard spin-selective pulses in the presence of longitudinal couplings, approach I (long; sel). The numerical estimates of the minimum pulse durations $T^{*}($ num $)$ and $T_{\text {long }}^{*}($ num $)$ (c.f. Table II) required to approach the unitary bound with an error of less than $10^{-5}$ are determined by the intersections of the horizontal dashed line (corresponding to $\log \left(Q_{\max }-Q\right)=-5$ ) with the red (approach I (iso; sel)) and blue (approach I (long; sel)) curves. In addition to the idealized cases of hard selective pulses, optimization results for the more realistic case of non-selective pulses in the presence of isotropic couplings and different offset frequencies (c.f. Table IV in Appendix A) are represented by black diamonds, approach I (iso; non-sel). In panels B-E, only the case of vanishing couplings between next-nearest neighbors $\left(J_{k, k+2}=0\right)$ is considered for simplicity (c.f. Fig. 4).

ange solid curves) only slightly improves the achievable transfer efficiency in Fig. 5 E. However, in Fig. 5 F the additional degrees of freedom reduce the pulse sequence duration to achieve the unitary bound by $50 \%$ to only about $4.55 T^{*}($ num $)$. In the three-spin system of Fig. 5 B, the use of four $90^{\circ}\left(N_{p}=M+1\right)$ pulses only improves the transfer efficiency compared to sequences consisting of three $90^{\circ}$ pulses $\left(N_{p}=M\right)$ for pulse sequence durations that are longer than the time $T=1 / J_{\max }$ to reach the unitary bound.

\section{E. Approach II: $N_{B}$ blocks}

Finally, Fig. 5 also shows the results of block optimizations using approach II. Open and solid blue squares represent results of block optimizations without and with periods $\Delta_{k}^{i s o}$ of isotropic mixing, respectively (approach II (long) and approach II (iso/long)). Light blue squares connected by light blue lines correspond to the optimization of $N_{B}=4$ blocks, whereas dark blue squares connected by dark blue lines correspond to the optimization of $N_{B}=16$ blocks. In the considered symmetric spin chains, no (Fig. $5 \mathrm{~A}, \mathrm{~B}$ ) or relatively small (Fig. $5 \mathrm{C}$, D) gains are found compared to approach III $\left(N_{p}=M\right)$. However, in Fig. $5 \mathrm{E}$ and F, significantly improved performance is found using approach II compared to approach III. In comparison to the performance of 4 blocks, 16 blocks yield slightly larger quality factors $Q$. The linear coupling topology with unequal couplings (Fig. $2 \mathrm{E}$ ) is the only spin system of the spin topologies considered here, where the use of isotropic mixing periods in approach $I I$ was found to be beneficial (see Fig. 5 E). Here, even with only four blocks, approach II (iso/long) yields pulse sequences that closely approach the unitary bound near $T=2 J_{\max }^{-1}$ (which is about $2.8 T^{*}($ num $)$ ) whereas for the same time the four-block sequences without isotropic mixing reaches only about $80 \%$ of the unitary bound.

The optimized pulse sequences (with and without isotropic mixing periods) for topology $\mathrm{E}$ based on approach $I I$ with four blocks and for a pulse duration of $T=2.2 J_{\max }^{-1}$ (close to the minimum time of approach II to reach the unitary bound) are summarized in section II B of the Supplementary Material ${ }^{55}$.

\section{F. Analysis of pulses by time-frequency representations}

Approach I does not assume a restricted family of pulse sequences and provides maximum flexibility for pulse sequence optimization. This allows us to explore the physical limits of pulse performance and to establish benchmarks for the maximum achievable MaxQ efficiency as a function of pulse duration, as discussed above. However, the large number of available degrees of freedom generally results in quite complicated and rapidly oscillating pulse shapes (c.f. Fig. S1 in the Supplementary Material ${ }^{55}$ ) that are difficult to interpret. As shown 

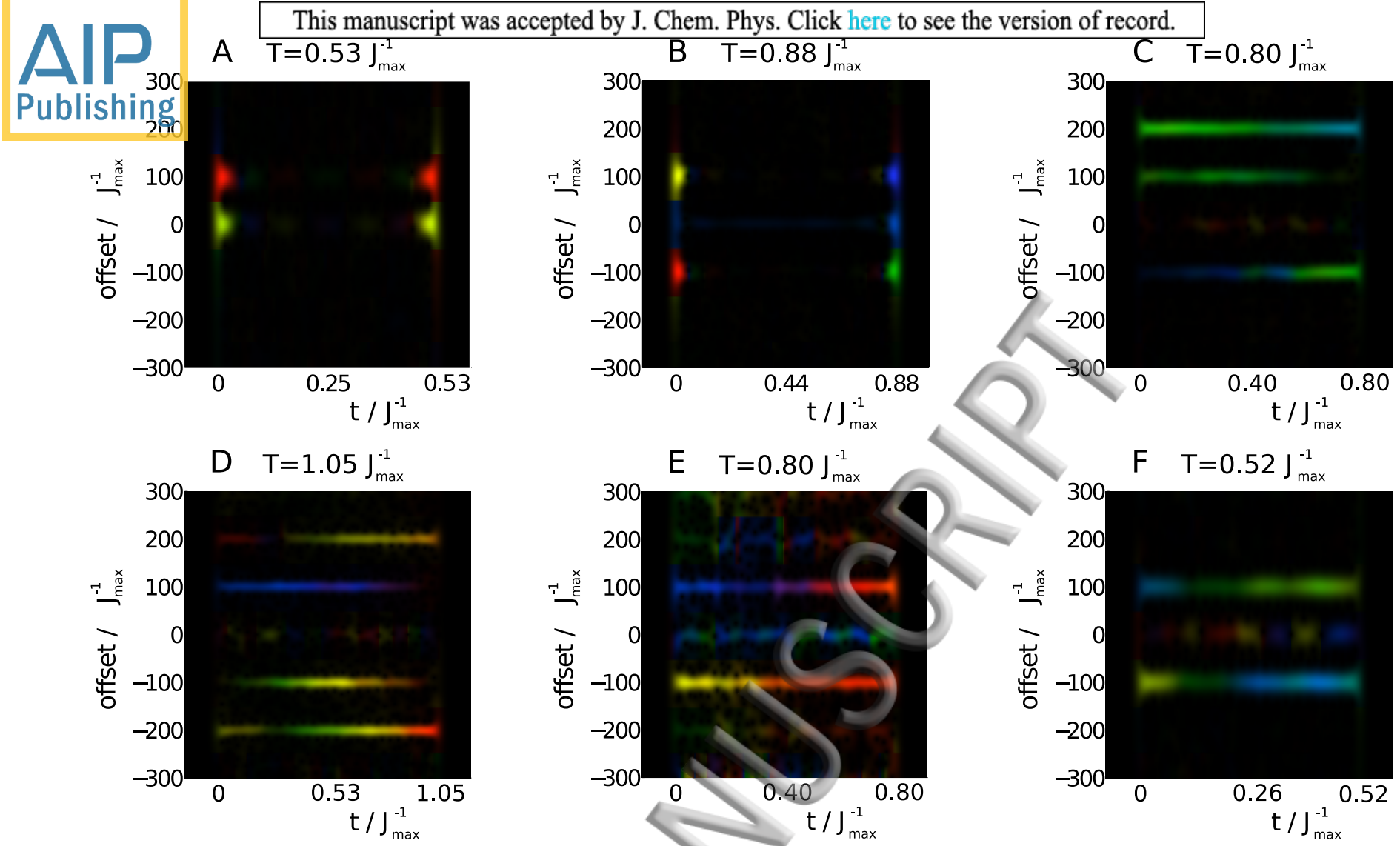

FIG. 7. Resonance-frequency-adapted phase-sensitive spectrograms ${ }^{48}$ of pulse sequences optimized using approach $I$ (iso; nonsel) with $J_{k, k+2}=0$ for the pulses shown in Fig. S1 in the Supplementary Material ${ }^{55}$. The normalized amplitude $A(t, \nu)$ is depicted as brightness and the phase $\varphi(t, \nu)$ is visualized as color (c.f. color bar Fig. 8 A). Panel B of the figure is reproduced with permission from J. Magn. Reson. 249, 64 (2014). Copyright 2014 Elsevier Inc.

TABLE III. Approximate flip angles $\alpha$ for each peak in the spectrogram. The flip angles are determined by integration over the $u_{x}$ amplitude along time for the crosssection pulse shapes shifted to $x$ phase. The integration limits are given as factors for the pulse duration $T$.

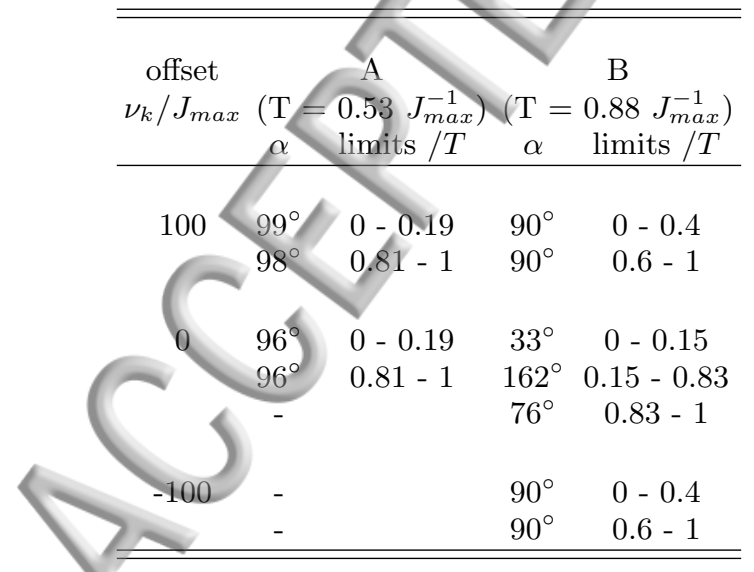

previously $^{48}$, a joint time-frequency analysis of pulses can provide a more intuitive and intelligible spectrogram representation of shaped pulses. Fig. S2 in the Supple- mentary Material ${ }^{55}$ shows representative absolute value spectrograms of pulses that were optimized for the spin systems summarized in Fig. 2 using approach I (iso; nonsel). Since the non-selective optimizations with approach $I$ (iso; non-sel) do not achieve $T^{*}$ (num) determined with approach I (iso; sel) (c.f. section II B), non-selective sequences which closely approach $Q_{\max }$ with $T \gtrsim T^{*}$ are chosen for the spectrogram representations of spin systems C-F (c.f. Fig. 2). For coupling topologies A and B, pulses with duration $T \approx T_{\text {long }}^{*}$ were chosen, although these pulses were optimized using approach I (iso; nonsel) in the limit of isotropic coupling and non-selective pulses. For $T \approx T_{\text {long }}^{*}$ interesting pulse shapes could be identified and analyzed. The spectrogram representation provides detailed information about the time-frequency structure of the pulses, which is not obvious in the temporal representation. In addition, Fig. 7 depicts corresponding resonance-frequency-adapted phase-sensitive spectrograms ${ }^{48}$. Although the pulse shapes were not restricted, the optimized pulses contain almost exclusively frequency components close to the offset frequencies $\nu_{k}$ of these spin systems. (This property is also found for shorter and longer pulse durations.) In Fig. $8 \mathrm{~A}$ and B, cross sections of Fig. $7 \mathrm{~A}$ and $\mathrm{B}$ are shown at the offsets 


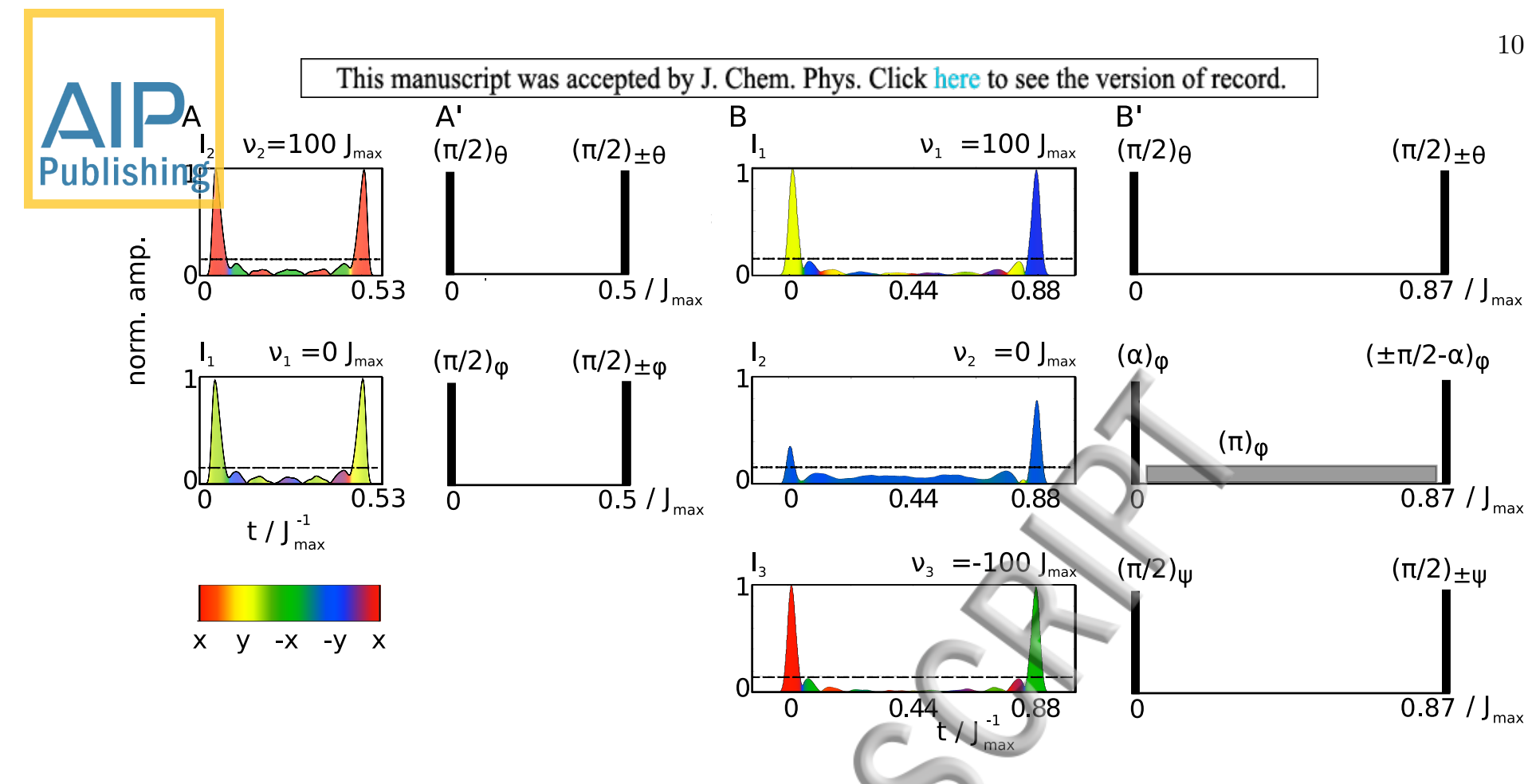

FIG. 8. The first and third columns show the cross sections of the phase-sensitive two-dimensional spectrogram representation (Fig. 7, approach I (iso; non-sel)) at the resonance frequencies of each spin (different rows) in systems A and B. The horizontal dashed lines visualize the threshold of $0.15 A_{\max }$ for the determination of the effective flip angle by integration of the amplitude over time. The areas of the individual peaks multiplied with the maximum amplitude $A_{\max }\left(A_{\max }(A)=135.9 J_{\max }\right.$, $\left.A_{\max }(B)=36.7 J_{\max }\right)$ correspond to the effective flip angles with the assumption of constant phase. The phase $\varphi(t, \nu)$ is color coded. The second and fourth columns $\left(A^{\prime}, B^{\prime}\right)$ show the schematic representation of the analytically derived, globally optimal pulse sequences based on the time-optimal (system A: $T_{\text {long }}^{*}($ ana $)=1 /(2 J)$, system B: $T_{\text {long }}^{*}($ ana $\left.)=\sqrt{3} /(2 J)\right)$ sequences for the generation of effective bilinear and trilinear coupling terms in system A and B in the longitudinal coupling limit (approach $I$ (long; sel) ). $\theta, \varphi$ and $\psi$ are arbitrary phase angles and describe the relationships of the pulse phases to each other. $\alpha$ is an arbitrary flip angle. Column B of the figure is reproduced with permission from J. Magn. Reson. 249, 64 (2014). Copyright 2014 Elsevier Inc.

$\nu_{k}$.

For system A (two-spin system) the optimized pulse sequence corresponds to the well-known approach shown in Fig. $8 \mathrm{~A}^{\prime}$ for longitudinal coupling. Two $90^{\circ}$ pulses that simultaneously act on both spins are separated by a delay of about $1 /(2 J)$. For each spin, the phases of the first and last pulses are identical (c.f. section III C approach $I I I$ and Fig. $8 \mathrm{~A}$ and $\left.\mathrm{A}^{\prime}\right)^{4,6}$.

Surprisingly, the shortest numerically optimized sequence using approach I (iso; non-sel) that closely approached the unitary bound had a duration of only $0.47 / J$ when starting with random initial pulses, indicating that it is possible to achieve MaxQ excitation in a duration that is shorter than $0.50 / J$. This numerical finding motivated a more detailed theoretical analysis of the minimum transfer time.

This analysis showed that it is in fact possible to achieve full double-quantum excitation in a duration of only $T^{*}($ ana $)=0.25 / J$ (c.f. Eq. (B16) in Appendix B and section III C of the Supplementary Material) in the limit of hard spin-selective pulses with negligible duration and using a period of isotropic mixing, which to the best of our knowledge has not been realized before. A detailed theoretical analysis of this novel transfer scheme is given

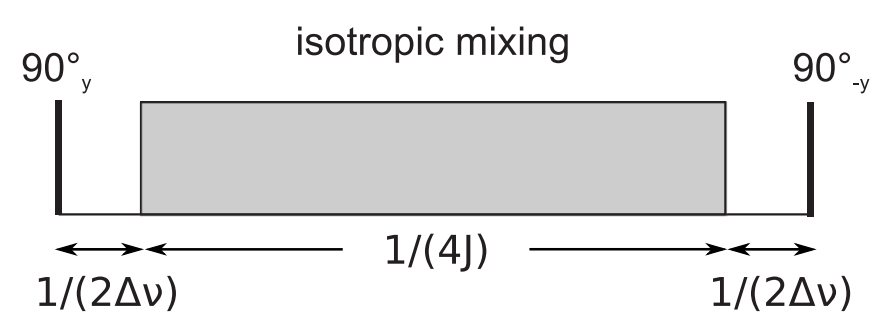

FIG. 9. Schematic representation of the analytically derived pulse sequence for the generation of $\operatorname{MaxQ}$ coherence in a two-spin system with isotropic mixing based on non-selective pulses. The $I_{1 x}-I_{2 x}$ state which is necessary for the generation of double-quantum coherence is achieved by a delay of $1 /(2 \Delta \nu)$ subsequent to the initial $90_{y}^{\circ}$ pulse.

in Appendix B. It is based on the generation of the initial state $I_{1 x}-I_{2 x}$ before the isotropic mixing period, which can also be achieved by a non-selective pulse followed by a delay of duration $1 /(2 \Delta \nu)$ (c.f. Fig. 9), assuming the first spin to be on resonance (c.f. Table I).

However, as discussed above, in this case the optimization algorithm based on non-selective pulses is prone to be trapped in local maxima, which appears to prevent the ab-initio optimization of the optimal isotropic mixing se- 
1 quence with $T^{*}($ ana $)=0.25 / J_{\max }$. However, numerical optimizations based on the spin-selective rf Hamiltonian Publishiling. $(7))^{38}$ were able to closely approach $T^{*}($ ana $)$, see solid red curve in Fig. 6 A. The numerically derived TOP curves for hard spin-selective pulses in the presence of longitudinal and isotropic couplings are shown in Fig. 6 .

Superficially, also the sequence optimized using approach I (iso; non-sel) for system B (three-spin chain) with $T \approx T_{\text {long }}^{*}$ appears to essentially consist of strong pulses applied at the beginning and at the end of the sequence, separated by a delay (c.f. Fig. 8 B). However, a closer inspection reveals that in fact an entirely different mechanism of maximum quantum excitation is exploited by this sequence. Note that in the conventional approach it would be mandatory to excite the second spin of the three-spin chain, because only this spin is coupled to both of the remaining spins of the system. The optimum duration of the conventional pulse sequence (approach III, $N_{p}=2$ ) is $0.5 J_{\max }^{-1}$ and only achieves a maximum efficiency of 0.2041 (c.f. red curve in Fig. 4). However, the shaped pulse optimized for system B has a significantly longer duration of $0.88 J_{\max }^{-1}$ and is able to closely approach the unitary bound of $\sqrt{3 / 8}=0.6124$ (c.f. Fig. 5 and Table II). Hence the optimized sequence is a factor of three (c.f. Appendix C and Fig. 10) more efficient than the conventional approach! In order to analyze this superior performance of the optimized pulse in more detail, it is helpful to inspect frequency-selective cross sections of the two-dimensional spectrogram representation ${ }^{48}$, which represent the individual pulse sequences that are experienced by each of the three spins (c.f. Fig. 8 B). In contrast to the first and third spin, the second spin is irradiated with a small but significant rf amplitude of constant phase essentially during the entire duration of the pulse sequence. This is reminiscent of analytically derived time-optimal pulse sequences for the creation of trilinear effective Hamiltonians in three-spin chains corresponding to system $\mathrm{B}$ with $J_{13}=0$ in the longitudinal coupling limit, so-called geodesic sequences ${ }^{26,27}$. This observation motivated a more detailed analysis of the optimal excitation of triple-quantum coherence in the threespin chain. As shown in Appendix C, it is possible to analytically derive the globally optimal pulse sequence with longitudinal coupling Hamiltonian based on the timeoptimal sequence ${ }^{27}$ for the generation of an effective trilinear coupling Hamiltonian. Fig. 8 B shows a schematic representation of the optimal geodesic sequence of duration $T_{\text {long }}^{*}($ ana $)=\sqrt{3} /\left(2 J_{\max }\right) \approx 0.87 / J_{\max }$ with an amplitude of $J_{\max } / \sqrt{3}$ of the weak pulse that is applied to the second spin. As shown in Figs. 7, $8 \mathrm{~B}$ and in Tables III and S4 of the Supplementary Material ${ }^{55}$, the flip angles, relative phases and the duration $T_{\text {long }}^{*}($ num $)$ closely match the analytically derived time-optimal geodesic solution of Fig. 8 B' (maximum deviation of flip angles $0.5 \%$, c.f. section VI of the Supplementary Material ${ }^{55}$ ). Fig. 10 shows the efficiency of MaxQ excitation for system $\mathrm{B}$ by representing the operator $I_{1}^{+} I_{2}^{+} I_{3}^{+}$using the
DROPS (discrete representation of operators for spin systems $)^{57}$ visualization. The Figure was created using the SpinDrops $\mathrm{app}^{58}$. As version 1.2 of SpinDrops assumes ideal pulses with negligible coupling evolution during the pulses, the long, weak $I_{2}$-selective pulse of Fig. $8 \mathrm{~B}$ was approximated using a DANTE-type sequence ${ }^{26}$ consisting of pulses with small flip angles $\left(5.6^{\circ}\right)$ separated by delays $\left(\sqrt{3} /\left(32 \cdot 2 J_{\max }\right) \approx 0.027 / J_{\max }\right)$. The conventional sequence (c.f. Fig. $10 \mathrm{~A}$ ) reaches its maximum performance of $33 \% Q_{\max }$ for $T=0.5 / J_{\max }$ and shows a decrease in the transfer efficiency for $T>0.5 / J_{\max }$ as described in section III B of the Supplementary Material ${ }^{55}$. In contrast, the geodesic sequence (c.f. Fig. 10 B) achieves $49 \% Q_{\max }$ for a pulse sequence duration of $T=0.5 / J_{\max }$ which corresponds to $146 \%$ of the transfer efficiency achieved by the conventional sequence. For the analytically derived minimal time $T_{\text {long }}^{*}($ ana $)=\sqrt{3} /\left(2 J_{\max }\right)$, the efficiency of the geodesic pulse sequence approaches the unitary bound of $\sqrt{3 / 8}$ in the longitudinal coupling limit. Note that in the isotropic mixing case the unitary bound is already approached for $T^{*}($ num $)=$ $0.57 J_{\max }^{-1}$. Therefore, we can provide a shaped pulse in the isotropic mixing limit which is not only a factor of three more efficient (c.f. section III B of the Supplementary Material ${ }^{55}$ and Table II) but also only marginally longer $\left(T^{*}(\right.$ num $\left.)=0.57 J_{\max }^{-1}\right)$ than the conventional approach based on two $90^{\circ}$ pulses $\left(T=0.50 J_{\max }^{-1}\right)$ ! For the remaining spin systems $\mathrm{C}-\mathrm{F}$, the numerically found pulses are very complex and have relatively high rf amplitudes. The spectrogram representations of these nonselective pulses (c.f. Figs. 7 and S2 in the Supplementary Material ${ }^{55}$ ) show that the sequences cannot be interpreted in terms of pulses and delays. Instead, the pulses consist of spin-selective ramped irradiation periods that often have constant rf phases.

Superficially, these pulse schemes are reminiscent of ramped adiabatic irradiation schemes ${ }^{59,60}$, but a more detailed analysis is beyond the scope of the present manuscript.

\section{EXPERIMENTS}

For an experimental demonstration of optimized MaxQ excitation sequences, we chose the ${ }^{1} \mathrm{H}$ spin system of 2,3-dibromopropionic acid (c.f. Fig. $3 \mathrm{C}$ ) dissolved in $\mathrm{CDCl}_{3}{ }^{56}$. The experiments were performed at room temperature using a Bruker Avance $600 \mathrm{MHz}$ spectrometer. The experimentally determined coupling constants are $J_{12}=11.4 \mathrm{~Hz}, J_{23}=4.4 \mathrm{~Hz}$, and $J_{13}=-10.15 \mathrm{~Hz}$. A Shigemi tube (magnetic susceptibility matched for $\mathrm{CDCl}_{3}$ ) with a diameter of $5 \mathrm{~mm}$ was used to achieve a rf field with high homogeneity.

In the experiments, spin 1 was set on resonance $\left(\nu_{1}=0\right)$, and the offsets for spins 2 and 3 were $347 \mathrm{~Hz}$ and -130 $\mathrm{Hz}$, respectively. After the excitation of MaxQ coherence of order three, other coherences were eliminated using a six-step phase cycling procedure. In all experiments, the 

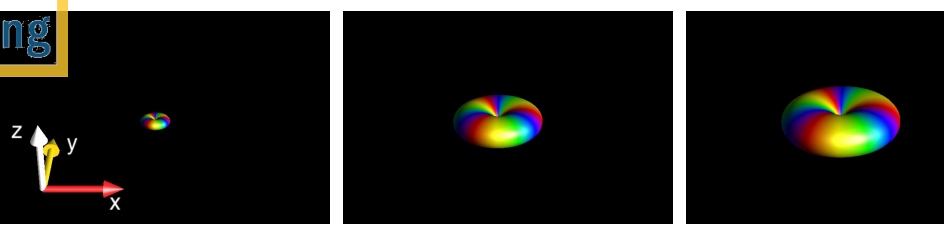

B
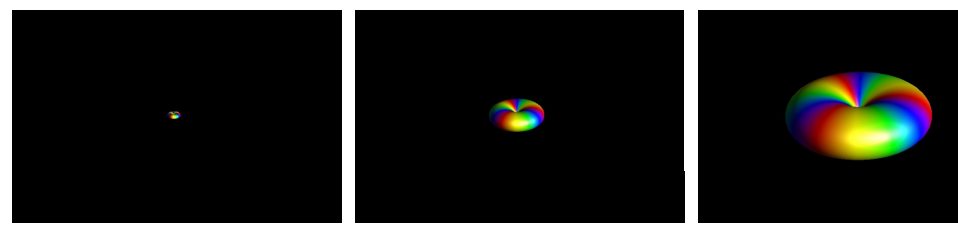

$$
\mathrm{T}=0.17 / \mathrm{J}^{-1} \max T=0.33 / \mathrm{J}^{-1} \max T=0.50 / \mathrm{J}^{-1} \mathrm{~m}
$$

FIG. 10. Efficiency of MaxQ excitation for a system consisting of three weakly coupled spins with $J_{12}=J_{23}=J_{m a x}$ and $J_{13}=0$ as a function of sequence duration for (A) the standard $90^{\circ}-\Delta-90^{\circ}$ sequence (c.f. Fig. 1 D) and (B) the geodesic pulse sequence (c.f. Fig. 8 B'). The operator $I_{1}^{+} I_{2}^{+} I_{3}^{+}$is visualized as donut-shaped objects using the DROPS representation ${ }^{57}$ (a generalized Wigner representation for coupled spin systems). The relative sizes of the shapes correspond to the relative amplitudes (MaxQ efficiencies) of $I_{1}^{+} I_{2}^{+} I_{3}^{+}$created by the different sequences and pulse durations. The color reflects a complex phase factor $\mathrm{e}^{\mathrm{i} \Phi}$ as defined by the color bar in Fig. 8, where $\mathrm{x}, \mathrm{y},-\mathrm{x}$ and $-\mathrm{y}$ correspond to $\Phi=0$ (red), $\pi / 2$ (yellow), $\pi$ (green) and $3 \pi / 2$ (blue), respectively. The figure was created using the SpinDrops app ${ }^{58}$.

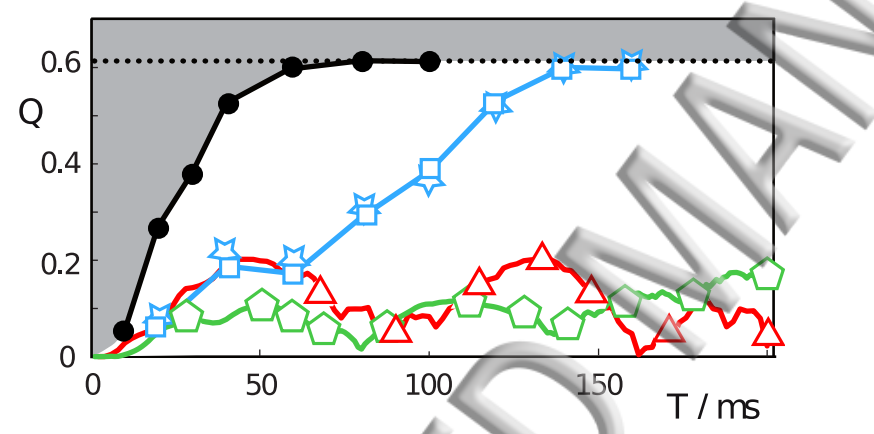

FIG. 11. Experimental and theoretical MaxQ excitation efficiencies $\mathrm{Q}$ of conventional and optimized pulse sequences for the ${ }^{1} \mathrm{H}$ spin system of 2,3-dibromopropionic acid (Fig. $3 \mathrm{C}$ ) with $J_{12}=11.4 \mathrm{~Hz}, J_{23}=4.4 \mathrm{~Hz}$, and $J_{13}=-10.15 \mathrm{~Hz}$ for pulse sequence durations $0 \leq T \leq 200 \mathrm{~ms}=2.28 / J_{\max }$. Red triangles and curves represent experimental and simulated $Q$ values for the simple standard sequence consisting of two $90^{\circ}$ pulses, respectively. Green pentagons and curves $(-\cdot \cdot)$ show the experimental and theoretical performance of optimized pulse sequences using approach III consisting of three $90^{\circ}$ pulses separated by optimal delays. Light blue stars and open squares (which are connected by straight lines to guide the eye) represent experimental and simulated results of $N_{B}=4$ blocks (approach II (long) without periods of isotropic mixing).

same sequence of $90_{x}^{\circ}-\tau-180_{-x}^{\circ}-\tau$ was used to convert MaxQ coherence to detectable single quantum coherence (c.f. section II D of the Supplementary Material ${ }^{55}$ ).

Fig. 11 shows the experimental excitation efficiencies and the corresponding theoretical curves. Although the ex- perimental relative coupling constants are similar to the ideal case shown in Figs. 2 F, 4 F and 5 F, we recalculated the TOP curve (using approach I (iso; non-sel)) and the maximum excitation efficiency for approaches II (long) and $I I I$ for the actual experimental coupling constants. A reasonable match is found between simulations and experiments. The pulse sequence consisting of two $90^{\circ}$ pulses performs better than the sequence with three $90^{\circ}$ pulses for the experimental range of pulse durations. But in agreement with Figs. $4 \mathrm{~F}$ and $5 \mathrm{~F}$ simulations show that the optimized three $90^{\circ}$ pulse sequence exceeds the quality factor achieved by two $90^{\circ}$ pulses considerably for longer pulse durations ( $T=250 \mathrm{~ms}$, data not shown). The minimum time to reach the unitary bound of MaxQ excitation for the block optimization (approach II (long)) is about $0.14 \mathrm{~s}$. This is about $88 \%$ shorter than the time needed using approach $I I I\left(T_{\min }=1.15 \mathrm{~s}\right.$, determined by numerical simulations). A factor of about three larger MaxQ amplitude is obtained compared to conventional approaches based on $90^{\circ}$ pulses.

\section{CONCLUSION}

In this paper, we explored the physical limits of the efficiency of maximum-quantum (MaxQ) excitation in model spin systems with defined coupling constants and offset frequencies. The detailed study of this topic opened a surprisingly rich and multifaceted field of research with many interesting aspects. For six model spin systems, the maximum amplitude of MaxQ coherence was determined using the general unitary bound of spin dynamics ${ }^{18-24}$. 
Theshortest possible duration $T^{*}$ to reach this bound wasnum erically explored using the optimal-control based Publi§aidngof arbitrary pulse shapes (approach I). This approach also allowed us to find time-optimal pulse (TOP) curves $^{25,38}$, which provide the maximum MaxQ amplitude for durations that are smaller than $T^{*}$. The TOP curves were obtained by optimizing the MaxQ transfer efficiency for a number of pulse sequence durations $T$ using approach $I$. As long as the offset frequencies are sufficiently separated $\left(\left|\Delta \nu_{\min }\right| \gg\left|J_{\max }\right|\right.$, where $\left|\Delta \nu_{\min }\right|$ is the minimum difference of offset frequencies), the TOP curves are independent of the specific chemical shifts and hence provide a general benchmark for the assessment of pulse sequences. However, the optimized pulse shapes differ for different offset frequencies and it is desirable to find robust offset-independent sequences that are also simple to implement. We therefore considered two different types of simple sequences. In approach II, we studied sequences consisting only of well defined broadband building blocks (hard pulses of arbitrary flip angle and phase, longitudinal coupling evolution and optional periods of isotropic mixing). In approach III, we explored simple pulse sequences consisting only of hard $90^{\circ}$ pulses with phases $x$ or $y$ and delays. The pulse sequences resulting from approaches $I I$ as well as $I I I$ can be made broadband by inserting $180^{\circ}$ refocusing pulses in the delays. It was found that approaches $I I$ and $I I I$ provide practical broadband sequences which closely approach the unitary bound. However, the sequence durations of approach $I I I$ to reach the unitary bound are up to a factor of 2.2 longer than the minimum time $T^{*}$ (num) for the case of chains consisting of up to five spins (Figs. $4 \mathrm{~A}-\mathrm{D}$ and $5 \mathrm{~A}-\mathrm{D}$ ). Large gains in MaxQ excitation are found compared to simple sequences consisting of two $90^{\circ}$ pulses and a delay.

Based on the numerical results, a novel analytical transfer scheme for two-spin systems was found. The application of isotropic mixing and hard spin-selective pulses allows for double-quantum coherence generation in twospin systems that is twice as fast as conventional approaches based on non-selective pulses and a delay.

The complicated pulse shapes resulting from approach $I$ were studied using short-time Fourier transform spectrograms ${ }^{48}$. In particular for the case of three-spin chains, this joint time-frequency representation provided valuable insight into the mode of action of the numerically optimized pulses. This allowed us to identify the general family of globally optimal geodesic pulse sequences for MaxQ excitation in the limit of longitudinal couplings. These geodesic sequences rely on a well defined slow rotation of the center spin in addition to hard pulses at the beginning and at the end. In practice, this can be implemented by a selective weak pulse applied to the central spin. Geodesic sequences that are robust to variations in chemical shifts can be constructed by implementing the slow rotation by a DANTE-type sequence ${ }^{61}$ based on (band-selective) small flip angle pulses and delays in combination with broadband refocusing pulses ${ }^{26}$.
For systems with nearest-neighbor couplings and spin chains with more than three coupled spins, a novel class of MaxQ excitation sequences based on ramped, selective pulses was found.

The optimal excitation of maximum quantum coherence based on approach $I I$ and $I I I$ was experimentally demonstrated for the case of 2,3-dibromopropionic acid. A reasonable match was found between the simulated and experimental MaxQ excitation efficiencies as a function of pulse duration. Compared to the best sequences consisting only of two $90^{\circ}$ pulses, a sensitivity gain of about a factor of three was experimentally achieved.

The unitary bounds summarized in Table II (and Figs. 4 and 5) correspond to the case of spin systems without permutation symmetry. This applies to the spin systems that were considered here for the optimizations using approach $I$, where all spins were assumed to be selectively addressable, either by selective pulses $\left(\mathcal{H}_{r f}^{\text {sel }} \neq 0\right)$ or based on different offset frequencies $\left(\mathcal{H}_{o f f} \neq 0\right)$, c.f. Table IV in Appendix A. For spin systems with magnetically equivalent spins, such as in $\mathrm{AX}_{2}$, $\mathrm{AX}_{3}, \mathrm{~A}_{2} \mathrm{X}_{2}$ spin systems etc., the unitary bounds can be further tightened due to the presence of permutation symmetry $19,20,22,24$.

Here, we focused on the optimization of experiments for-specific spin systems with well defined coupling topologies, coupling constants and offset frequencies. It is also possible to optimize robust pulse sequences for a desired range of coupling constants, chemical shifts and coupling topologies that are of interest for a given application, such as the analysis of biofluids or mixtures of aromatic hydrocarbons ${ }^{8}$. Conversely, it is also possible to maximize MaxQ excitation selectively for a given set of spin systems while suppressing multiple quantum excitation for others in order to simplify spectra with overlapping signals.

In this paper the transfer from thermal equilibrium $\left(F_{z}\right)$ to MaxQ coherence $\left(P^{+}\right.$, c.f. Eq. $\left.(9)\right)$ was investigated. The reversed transfer from $P^{+}$to detectable single quantum coherence can be optimized using the same approach. The optimized $F_{z} \rightarrow P^{+}$sequences can be converted to $P^{+} \rightarrow F_{z}$ sequences by time and phase inversion. ${ }^{12}$ An additional $90_{y}^{\circ}$ pulse converts $F_{z}$ to $F_{x}$. However, the maximum efficiency ${ }^{19,20,22,24}$ for the transfer between the two non-Hermitian operators $P^{+}$and the detection operator $F^{-}$is larger than for the transfer between $P^{+}$and $F_{x}$ and hence it is expected that an additional gain in sensitivity by specifically optimizing sequences for the $P^{+} \rightarrow F^{-}$transfer is achievable.

In the examples considered here, relaxation effects were neglected, which is justified for applications to small molecules, where relaxation times are longer than the duration of multiple quantum excitation. This is the case for 2,3-dibromopropionic acid ${ }^{62,63}$ with $J_{\max }=11.4 \mathrm{~Hz}$ shown in Fig. 11, where the optimized, experimentally implemented MaxQ excitation sequences based on block optimization (approach II) have a duration of about $150 \mathrm{~ms}$. For applications (e.g. to proteins), where relax- 
1 ation is not negligible, it is possible to include relaxation effects in the pulse sequence optimization ${ }^{29,45}$.

Publishting oresented approach for efficient excitation of MaxQ coherence can also be applied to study the efficient generation of multiple quantum coherence of lower order.

It is expected that the presented methods and benchmarks will help to develop significantly improved pulse sequences for practical applications.

\section{ACKNOWLEDGMENTS}

We thank N. Chandrakumar and A. Bax for helpful discussions and comments. S.J.G. acknowledges support from the DFG (Gl 203/7-2), SFB 631. Experiments were performed at the Bavarian NMR center at TU München.

\section{APPENDIX}

Appendix A: Definition of the studied limiting cases and of the minimal times $T^{*}$ and $T_{\text {long }}^{*}$

In order to see general trends in the maximum possible performance of MaxQ experiments, it is useful to consider characteristic limiting cases, c.f. Table IV. This provides a level of abstraction that allows us to establish generic benchmarks that are applicable for a large class of experimentally relevant spin systems and pulse sequences.

Possible criteria for the classification of spin systems and the corresponding pulse sequences are

(a) homonuclear versus heteronuclear systems,

(b) weak versus strong coupling,

(c) longitudinal versus isotropic coupling terms,

(d) non-selective versus spin-selective pulses.

(a) Here, for simplicity we focus on the case of homonuclear spin systems. In heteronuclear/spin systems, the thermal equilibrium density operator depends on the relative size of the the gyromagnetic ratios of the spins and is not simply proportional to $F_{z}$ as in the homonuclear case, c.f. Eq. (8). Hence the MaxQ excitation efficiency depends on the specific combination of hetero spins. Furthermore, the scaling factor $J_{k l}^{e f f, i s o} / J_{k l}{ }^{52}$ is limited by $1 / 3$ for the effective coupling constants in heteronuclear isotropic mixing sequences ${ }^{52}$, whereas $J_{k l}^{e f f, i s o} / J_{k l} \leq 1$ in homonuclear experiments considered here.

(b) For simplicity, we only consider weakly coupled (homonuclear) spin systems, where for all spin pairs $I_{k}$ and $I_{l}$ the coupling constants $J_{k l}$ are much smaller than the offset differences of the spins, i.e. $\left|J_{k l}\right| \ll\left|\nu_{k}-\nu_{l}\right|$.

(c) It is important to note that even for weakly coupled homonuclear spins systems, in general the full isotropic coupling terms $\mathcal{H}_{c}^{\text {iso }}$ of Eq. (4) needs to be considered to correctly describe the time evolution under an arbitrary pulse sequence ${ }^{3}$. In particular it is possible to suppress the offset terms $\mathcal{H}_{\text {off }}$ of Eq. (3) and to recover the full isotropic coupling terms $\mathcal{H}_{c}^{\text {iso }}$ (Eq. 4) with the help of broadband isotropic mixing sequences ${ }^{52,64}$. If the maximum available rf amplitude $u_{\max }$ is larger than the offsets $\nu_{k}$, scaling factors $J_{k l}^{e f f, i s o} / J_{k l}$ of about 1 can be approached, i.e. $J_{k l}^{e f f, i s o} \approx J_{k l}$. However, during delays or periods of weak rf irradiation, the correct spin dynamics is closely approximated by considering only the longitudinal coupling terms $\mathcal{H}_{c}^{\text {long }}$ (c.f. Eq. (5)) in the weak coupling limit.

(d) The sequence families considered in approaches $I I$ and III consist only of idealized broadband, non-selective pulse sequence elements such as hard pulses, periods of longitudinal coupling evolution (corresponding to delays with refocusing pulses) and periods of isotropic mixing (corresponding to broadband isotropic mixing sequences). In the theoretical analysis of these sequences, it is useful to consider the limit of short non-selective pulses, where the duration of non-selective pulses (on the order of $u_{\text {max }}^{-1}$ ) is negligible compared to the overall pulse sequence duration $T$ (on the order of $J_{\max }^{-1}$ ), which is valid if $u_{\max } \gg J_{\max }$.

In the most general case of approach $I$, pulse sequences are not restricted to combinations of hard, offsetindependent pulses. Using frequency-selective weak irradiation, it is possible to realize spin-selective rotations. In this case, it is useful to consider also the limit of hard spin-selective pulses with negligible duration (duration on the order of $\left|\nu_{k}-\nu_{l}\right|^{-1}$ ), which can be approached if $\left|\nu_{k}-\nu_{l}\right| \gg J_{\max }$. Relatively fast spin-selective rotations can be implemented by shaped pulses or by simple combinations of non-selective pulses and delays (c.f. Fig. 9). We call this the limit of hard selective pulses. In this limit, the theoretical analysis of homonuclear pulse sequences can be further simplified by describing the spin system in a multiple-rotating frame (similarly as in the case of heteronuclear spin systems). Hence, the offsetterm $\mathcal{H}_{\text {off }}$ (c.f. Eq. (3)) of the free evolution Hamilton $\mathcal{H}_{0}$ can be neglected when optimizing idealized sequences to explore numerically the minimum time for MaxQ excitation (see Table IV).

Note that the universal bound $Q_{\max }$ for the maximum possible transfer amplitude for a given initial and final state is independent of the experimental setting. However, the minimum time to achieve $Q_{\max }$ depends on the parameters of the spin system and on the considered limiting case. For simplicity, here we distinguish the minimum times for the following two cases:

$T^{*}$ denotes the minimal time to achieve the unitary bound for a homonuclear spin system in the weak coupling limit, but assuming the ability to exploit the full isotropic coupling term $\mathcal{H}_{c}^{\text {iso }}$ and the limit of hard selective pulses as defined above. This corresponds to situations, where $u_{\max } \gg\left|\nu_{k}-\nu_{l}\right| \gg J_{\max }$.

$T_{\text {long }}^{*}$ denotes the minimal time to achieve the unitary bound for a homonuclear spin system in the weak coupling limit, assuming the limit where only longitudinal coupling terms $\mathcal{H}_{c}^{\text {long }}$ can be exploited. Furthermore, it is assumed that hard spin-selective pulses can be short 
1 eneng to be negligible compared to the overall pulse equence duration (hard selective pulses). This correPublisplahag to situations, where $\left|\nu_{k}-\nu_{l}\right| \gg u_{\max } \gg J_{\max }$

For the sequence families in approaches $I, I I$, and $I I I$, Table IV summarizes all combinations of coupling tensors (isotropic versus longitudinal) and pulse selectivity considered in the text. For each of these cases, the used terms in the Hamiltonian are indicated by a bullet. The last column summarizes for which cases the minimum times to excite MaxQ coherence were numerically explored and defines the corresponding labels used in the text. Note that the limiting cases of approach I (iso; non-sel) with $\left|\nu_{k}-\nu_{l}\right| \gg J_{\max }$ and approach I (iso; sel) with hard spin-selective pulses are expected to require the same minimum time $T^{*}$. If this was not the case in the numerical optimizations (c.f. section II B) the smaller of these two values is given in Table II as our best numerical estimates $T^{*}$ (num) of $T^{*}$. As shown in Fig. 6, the smaller minimum times were always found for the case of hard selective pulses (red circles), whereas longer times were found for the case of non-selective pulses (black open diamonds). Similarly, the limiting cases of approach I (long; non-sel) and approach I (long; sel) are expected to require the same minimum time $T_{\text {long }}^{*}$. For completeness, table IV also summarizes the considered Hamiltonian terms in the cases of isotropic and longitudinal couplings for which the theoretical limits $T^{*}($ ana $)$ and $T_{\text {long }}^{*}($ ana $)$ presented in Table II were derived analytically (c.f. sections IV and V of the Supplementary Material ${ }^{55}$.

\section{Appendix B: Time optimal generation of double-quantum coherence in a system consisting of two coupled spins}

In this section, we analytically derive the minimum times $T^{*}($ ana $)$ and $T_{\text {long }}^{*}($ ana $)$ to achieve the unitary bound $Q_{\max }=1 / \sqrt{2}$ (c.f. Table II) for the excitation of double-quantum coherence in a homonuclear system consisting of two spin $1 / 2$ particles. Assuming the limit of hard selective pulses with negligible duration, $T^{*}($ ana $)$ and $T_{\text {long }}^{*}$ (ana) correspond to the idealized cases of isotropic and longitudinal coupling terms $\left(H_{c}^{i s o}\right.$, Eq. (4) and $H_{c}^{\text {long }}$, Eq. (5)), respectively.

In order to derive $T^{*}($ ana $)$ and $T_{\text {long }}^{*}($ ana $)$, we first identify the general form of a propagator $U$ that achieves the unitary bound for the desired transfer and we specify the family of effective Hamiltonians creating propagators of the desired form. Finally, the minimum time to create the propagator $U$ can be derived based on the results of Khaneja et al. ${ }^{28,65,66}$

The transfer efficiency $Q$ from an initial state $A$ to a target state $C$ by the propagator $U$ is defined as ${ }^{19,20,22,24}$

$$
\begin{aligned}
Q & =\frac{\left|\left\langle U A U^{\dagger} \mid C\right\rangle\right|}{\|A\| \cdot\|C\|} \\
& =\frac{\left|\operatorname{Tr}\left(U A^{\dagger} U^{\dagger} C\right)\right|}{\sqrt{\operatorname{Tr}\left(A^{\dagger} A\right)} \sqrt{\operatorname{Tr}\left(C^{\dagger} C\right)}},
\end{aligned}
$$

which reduces to Eq. (10) for the initial density operator $\rho(0)=A$, the final density operator $\rho(T)=U A U^{\dagger}$ and the target operator $C=P^{+}$.

The initial state $A=I_{1 z}+I_{2 z}$ corresponds to the thermal equilibrium operator for the case of a homonuclear spin system and the target state $C=I_{1}^{+} I_{2}^{+}$to doublequantum coherence with coherence order $p=+2$.

Explicit matrices for the operators $A$ and $C$ are given in section IV A of the Supplementary Material ${ }^{55}$. For these initial and final states and a general propagator represented by the unitary matrix

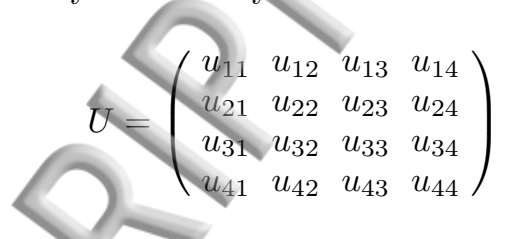

(with in general complex matrix elements $u_{k l}$ ), a straightforward calculation yields

$$
Q=\frac{1}{\sqrt{2}}\left|u_{11}^{*} u_{41}-u_{14}^{*} u_{44}\right| \text {. }
$$

Based on Eq. (B4) and the properties of unitary matrices, it can be shown (c.f. section IV B of the Supplementary Material $^{55}$ ) that the maximum transfer efficiency $Q$ is given by

$$
Q_{\max }=\frac{1}{\sqrt{2}}
$$

which corresponds to the unitary bound ${ }^{18-24}$ for this transfer (c.f. Table II). The most general form of a propagator achieving this bound is given by

$$
U^{o p t}=\left(\begin{array}{cccc}
\frac{1}{\sqrt{2}} e^{i r_{1}} & 0 & 0 & -\frac{1}{\sqrt{2}} e^{i r_{2}} \\
0 & u_{22} & u_{23} & 0 \\
0 & u_{32} & u_{33} & 0 \\
\frac{1}{\sqrt{2}} e^{i\left(r_{1}+r_{3}\right)} & 0 & 0 & \frac{1}{\sqrt{2}} e^{i\left(r_{2}+r_{3}\right)}
\end{array}\right)
$$

with arbitrary real numbers $r_{1}, r_{2}$, and $r_{3}$ and where the sub block consisting of the matrix elements $u_{22}, u_{23}, u_{32}$, and $u_{33}$ can be an arbitrary unitary $2 \times 2$ matrix (see section IV B of the Supplementary Material $\left.{ }^{55}\right)$. Hence, the minimum times $T^{*}($ ana $)$ and $T_{\text {long }}^{*}($ ana $)$ to achieve the unitary bound $Q_{\max }$ for the excitation of doublequantum coherence correspond to the minimum times to create a propagator of the form $U^{o p t}$ by a pulse sequence in the isotropic and longitudinal coupling case, respectively.

As shown in section IV $\mathrm{C}$ of the Supplementary Material ${ }^{55}$, the creation of $U^{o p t}$ takes the same amount of time as the creation of a propagator of the form

$$
U^{b i}=\exp \left\{-i \pi\left(c_{x x} I_{1 x} I_{2 x}+c_{y y} I_{1 y} I_{2 y}+c_{z z} I_{1 z} I_{2 z}\right\}\right.
$$

with $\left|c_{x x}-c_{y y}\right|=1$. $U^{b i}$ contains only the bilinear terms $I_{1 x} I_{2 x}, I_{1 y} I_{2 y}$, and $I_{1 z} I_{2 z}$ in the exponent and hence has the appropriate form to derive the minimal times 
V. Hamiltonian terms considered in different numerical and theoretical approaches. The Hamiltonian terms are Publisfingdoin the following equations of the main text: $\mathcal{H}_{c}^{\text {iso }}$ (Eq. (4)), $\mathcal{H}_{c}^{\text {long }}$ (Eq. (5)), $\mathcal{H}_{r f}^{\text {non-sel }}($ Eq. $(6)), \mathcal{H}_{r f}^{\text {sel }}($ Eq. $(7))$, and H. off (I. (3)). Numerical estimates $T^{*}\left(\right.$ num) for the minimal duration $T^{*}$ are provided both by approach $I$ (iso; non-sel or sel). Similarly, numerical estimates for $T_{\text {long }}^{*}$ are provided by approach I (long; non-sel or sel).

\begin{tabular}{|c|c|c|c|c|c|c|}
\hline sequence family & $\mathcal{H}_{c}^{i s o}$ & $\mathcal{H}_{c}^{\text {long }}$ & $\mathcal{H}_{r f}^{\text {non-sel }}$ & $\mathcal{H}_{r f}^{\text {sel }}$ & $\mathcal{H}_{\text {off }}$ & min. time \\
\hline approach I (iso; non-sel) & $\bullet$ & - & $\bullet$ & - & , & $T^{*}($ num $)$ \\
\hline approach I (iso; sel) & $\bullet$ & - & - & & & $T^{*}($ num $)$ \\
\hline theoretical limit (iso) & $\bullet$ & - & - & $\bullet$ & & \\
\hline approach I (long; non-sel) & - & $\bullet$ & $\bullet$ & - & & \\
\hline approach I (long; sel) & - & $\bullet$ & - & & & 7 \\
\hline theoretical limit (long) & - & $\bullet$ & - & & & \\
\hline approach II (iso/long) & • & & 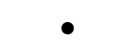 & & & \\
\hline approach II (long) & - & $\bullet$ & & & & \\
\hline approach III & - & - & $\bullet$ & & & - \\
\hline
\end{tabular}

$T^{*}($ ana $)$ and $T_{\text {long }}^{*}($ ana $)$ based on the approach of Yuan and Khaneja. ${ }^{28}$.

In their paper, Eq. (19) of Theorem 2 defines the minimum time $T$ required to generate a propagator $U$ using a constant Hamiltonian $\mathcal{H}_{c}$ by

$$
\begin{array}{ll} 
& \vec{\beta}_{\vec{n}} \prec_{s} \vec{\theta}^{\mathcal{H}_{c}} T \\
\text { with } & \vec{\beta}_{\vec{n}}=\vec{\theta}^{U}+2 \pi \vec{n}
\end{array}
$$

for either $\vec{n}=(0,0,0)$ or $\vec{n}=(-1,0,0)$, where $\vec{\theta}_{c}$ and $\overrightarrow{\theta^{U}}$ are the so-called canonical forms of the bilinear coefficients of the Hamiltonian $\mathcal{H}$ and the propagator $U$, respectively ${ }^{28}$. The symbol ' $\prec_{s}$ ' represents the operator of $s$-majorization and $\vec{\beta}$ is a real $s$-ordered vector of dimension three (see section IV D of the Supplementary Material $\left.^{55}\right)$.

For the longitudinal and isotropic coupling cases for $\vec{n}=(0,0,0)$, Eq. (B8) results in

$$
\vec{\beta}_{(0,0,0)}=\vec{\theta}^{\mathrm{U}^{b i}} \prec_{s} \vec{\theta}^{\mathcal{H}_{c}} T
$$

corresponding to the conditions

$$
\left(\begin{array}{c}
\theta_{1}^{U} \\
\theta_{2}^{U} \\
\theta_{3}^{U}
\end{array}\right) \prec_{s} 2 \pi J\left(\begin{array}{l}
1 \\
0 \\
0
\end{array}\right) T_{\text {long }}
$$

and

$$
\left(\begin{array}{c}
\theta_{1}^{U} \\
\theta_{2}^{U} \\
\theta_{3}^{U}
\end{array}\right) \prec_{s} 2 \pi J\left(\begin{array}{l}
1 \\
1 \\
1
\end{array}\right) T_{i s o},
$$

which are more restrictive than the conditions for $\vec{n}_{(-1,0,0)}$. The inequalities (4) in the paper by Yuan and Khaneja ${ }^{28}$, which describe the properties of $s$-majorized vectors, translate to

$$
\begin{aligned}
\theta_{1}^{U}+\theta_{2}^{U}+\left|\theta_{3}^{U}\right| & \leq 2 \pi J T_{\text {long }} \\
\text { and } \quad 2 \theta_{1}^{U}+2 \theta_{2}^{U} & \leq 8 \pi J T_{\text {iso. }} .
\end{aligned}
$$

As derived in section IV D of the Supplementary Material ${ }^{55}$, this implies that the minimum times $T_{\text {long }}^{*}($ ana $)$ and $T^{*}($ ana $)=T_{\text {iso }}^{*}$ are given by

$$
\begin{aligned}
& T_{\text {long }}^{*}(\text { ana })=\frac{1}{2 J} \\
& T^{*}(\text { ana })=\frac{1}{4 J},
\end{aligned}
$$

respectively.

The ideal pulse sequence for generating doublequantum coherence in the isotropic coupling limit is based on the conversion of the initial state $I_{1 x}-I_{2 x}$ to $2 I_{1 y} I_{2 z}-2 I_{1 z} I_{2 y}$ by an isotropic mixing period of duration $1 /\left(4 J_{\max }\right)$.

$$
\begin{aligned}
& \rho(0)=I_{1 z}+I_{2 z} \stackrel{90_{y}^{\circ}}{\longrightarrow} I_{1 x}+I_{2 x} \stackrel{180_{z}^{\circ}\left(I_{2}\right)}{\longrightarrow} I_{1 x}-I_{2 x} \\
& \stackrel{2 \pi J_{12}\left(I_{1 x} I_{2 x}+I_{1 y} I_{2 y}+I_{1 z} I_{2 z}\right) 1 /(4 J)}{\longrightarrow} 2 I_{1 y} I_{2 z}-2 I_{1 z} I_{2 y} \\
& \stackrel{180_{z}^{\circ}\left(I_{2}\right)}{\longrightarrow} 2 I_{1 y} I_{2 z}+2 I_{1 z} I_{2 y} \stackrel{90_{y}^{\circ}}{\longrightarrow}-2 I_{1 y} I_{2 x}-2 I_{1 x} I_{2 y}
\end{aligned}
$$

The operator $I_{1 x}-I_{2 x}$ is created from $I_{1 x}+I_{2 x}$ by an $I_{2}$-selective $180_{z}^{\circ}$ rotation, which can be realized by a delay (c.f. Fig. 9). Similarly, the operator $2 I_{1 y} I_{2 z}-2 I_{1 z} I_{2 y}$ (corresponding to zero-quantum coherence in the tilted frame with the $\mathrm{x}$ axis as quantization axis), which is created by the isotropic mixing period, can be transformed by an $I_{2}$-selective $180_{z}^{\circ}$ rotation to $2 I_{1 y} I_{2 z}+2 I_{1 z} I_{2 y}$ (corresponding to double-quantum coherence in the tilted frame with the $\mathrm{x}$ axis as quantization axis). A nonselective $90_{y}^{\circ}$ pulse finally results in $-2 I_{1 y} I_{2 x}-2 I_{1 x} I_{2 y}$ (corresponding to the desired double-quantum coherence in the rotation frame with quantization axis z). In the context of heteronuclear cross-polarization experiments, the creation of zero-quantum coherence by planar 
Publisdaing giously discussed ${ }^{67}$. However, for the heteronuclear case, a planar mixing period of duration $1 /(2 \mathrm{~J})$ would be required for the full conversion, whereas an isotropic mixing period of duration $1 /(4 J)$ is sufficient in the homonuclear case considered here (c.f. Fig. 9).

\section{Appendix C: Time optimal generation of triple-quantum coherence in a three-spin chain based on geodesic pulses}

Here we consider the case of a linear three-spin chain with only nearest-neighbor longitudinal couplings $J_{12}=J_{23}=J$ and $J_{13}=0$. According to Eq. (B1), the transfer efficiency from the initial state $A=I_{1 z}+I_{2 z}+I_{3 z}$ (corresponding to the thermal equilibrium operator for the case of a homonuclear spin system) to the target state $C=I_{1}^{+} I_{2}^{+} I_{3}^{+}$(corresponding to triple-quantum coherence with coherence order $p=+3$ ) by a unitary transformation $U$ is given by

$$
\begin{aligned}
Q & =\frac{1}{2 \sqrt{6}} \mid 3 u_{11}^{*} u_{81}+u_{12}^{*} u_{82}+u_{13}^{*} u_{83}-u_{14}^{*} u_{84} \\
& +u_{15}^{*} u_{85}-u_{16}^{*} u_{86}-u_{17}^{*} u_{87}-3 u_{18}^{*} u_{88} \mid,
\end{aligned}
$$

with the upper bound of (for details c.f. sections V A and V B of the Supplementary Material ${ }^{55}$ )

$$
Q_{\max }=\sqrt{\frac{3}{8}}
$$

The unitary operator $U$ achieving the maximal transfer has the general form

$$
U^{o p t}=\left(\begin{array}{ccccc}
\frac{1}{\sqrt{2}} e^{i r_{1}} & 0 & \ldots & 0 & \frac{1}{\sqrt{2}} e^{i r_{2}} \\
0 & u_{22} & \ldots & u_{27} & 0 \\
\vdots & \vdots & \ddots & \vdots & \vdots \\
0 & u_{72} & \ldots & u_{77} & 0 \\
-\frac{1}{\sqrt{2}} e^{-i r_{2}} & 0 & \ldots & 0 & \frac{1}{\sqrt{2}} e^{-i r_{1}}
\end{array}\right),
$$

with arbitrary real numbers $r_{1}$ and $r_{2}$ (c.f. section $\mathrm{V}$ B of the Supplementary Material ${ }^{55}$ ). In general, all $U^{t r i}=$

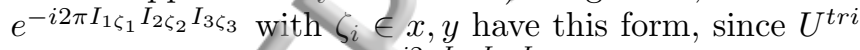
can be generated from $e^{-i 2 \pi I_{1 x} I_{2 x} I_{3 x}}$ by a unitary transformation with $e^{-i\left(\alpha_{1} I_{1 z}+\alpha_{2} I_{2 z}+\alpha_{3} I_{3 z}\right)}$ (c.f. section $\mathrm{V} \mathrm{C}$ of the Supplementary Material $\left.{ }^{55}\right)$.

Particularly, $U^{t r i}=e^{-i 2 \pi I_{1 y} I_{2 x} I_{3 y}}$ has this form and thus can achieve the maximal transfer efficiency. From previous work 26,27 , the time optimal sequence generating $U^{\text {tri }}$ for a linear three-spin chain in the longitudinal coupling limit with Hamiltonian $\mathcal{H}_{c}^{\text {long }}=2 \pi J\left(I_{1 z} I_{2 z}+\right.$ $\left.I_{2 z} I_{3 z}\right)$ is

$$
\begin{aligned}
& e^{-i \frac{\pi}{2}\left(I_{1 x}-I_{2 x}+I_{3 x}\right)} e^{T\left[-i 2 \pi J\left(I_{1 z} I_{2 z}+I_{2 z} I_{3 z}\right)+i \frac{\pi}{T} I_{2 x}\right]} \\
& e^{i \frac{\pi}{2}\left(I_{1 x}+I_{3 x}\right)},
\end{aligned}
$$

where $T=T_{\text {long }}^{*}($ ana $)=\sqrt{3} /(2 J)$ (c.f. section V D of the Supplementary Material $\left.{ }^{55}\right)$.
${ }^{1}$ M. H. Levitt, R. R. Ernst, Spin-pattern recognition in highresolution proton NMR spectroscopy, Chem. Phys. Lett. 100, 119-123 (1983).

${ }^{2}$ D. P. Weitekamp, J. R. Garbow, J. B. Murdoch, A. Pines, HighResolution NMR Spectra in Inhomogeneous Magnetic Fields: Application of Total Spin Coherence Transfer Echoes, J. Am. Chem. Soc. 103, 3578-3579 (1981).

${ }^{3}$ R. R. Ernst, G. Bodenhausen, A. Wokaun, Principles of Nuclear Magnetic Resonance in One and Two Dimensions, Oxford Science Publications, Clarendon Press, Oxford, 1987.

${ }^{4}$ W. P. Aue, E. Bartholdi, R. R. Ernst, Two-dimensional spectroscopy. Application to nuclear magnetic resonance. J. Chem. Phys. 64, 2229-2246 (1976).

${ }^{5}$ G. Bodenhausen, Multiple-quantum NMR, Prog. NMR Spectrosc. 14, 137-173 (1980).

${ }^{6}$ L. Braunschweiler, G. Bodenhausen, R. R. Ernst, Analysis of networks of coupled spins by multiple quantum NMR, Mol. Phys. 48, 535-560 (1983).

${ }^{7}$ M. Munowitz, A. Pines, Multiple-quantum nuclear magnetic resonance spectroscopy, Science 233, 525-531 (1986).

${ }^{8}$ G. N. M. Reddy, S. Caldarelli, Demixing of severely overlapping NMR spectra through multiple-quantum NMR, Anal. Chem. 82, 3266-3269 (2010).

${ }^{9}$ G. N. M. Reddy, S. Caldarelli, Maximum Quantum (MaxQ) NMR for the speciation of mixtures of phenolic molecules, Chem. Comm. 47, 4297-4299 (2011).

${ }^{10}$ G. N. M. Reddy, S. Caldarelli, Identification and quantification of EPA 16 priority polycyclic aromatic hydrocarbon pollutants by Maximum-Quantum NMR, Analyst 137, 741-746 (2012).

${ }^{11}$ G. N. M. Reddy, S. Caldarelli, Improved excitation uniformity in multiple-quantum NMR experiments of mixtures, Magn. Reson. Chem. 51, 240-244 (2013).

${ }^{12}$ M. H. Levitt, R. R. Ernst, Multiple-quantum excitation and spin topology filtration in high-resolution NMR, J. Chem. Phys. 83, 3297-3310 (1985)

${ }^{13}$ U. Piantini, O. W. Sørensen, R. R. Ernst, Multiple quantum filters for elucidating NMR coupling networks, J. Am. Chem. Soc., 104, 6800-6801 (1982).

${ }^{14}$ S. Saxena, J. H. Freed, Double quantum two-dimensional Fourier transform electron spin resonance: distance measurements, Chem. Phys. Lett. 251, 102-110 (1996).

${ }^{15} \mathrm{~S}$. Saxena, J. H. Freed, Theory of double quantum twodimensional electron spin resonance with application to distance measurements, J. Chem. Phys., 107, 1317-1340 (1997).

${ }^{16}$ P. P. Borbat, J. H. Freed, Multiple-quantum ESR and distance measurements, Chem. Phys. lett., 313, 145-154 (1999)

${ }^{17}$ A. Schweiger, G. Jeschke, Principles of Pulse Electron Paramagnetic Resonance, Oxford University Press, New York, 2001.

${ }^{18} \mathrm{O}$. W. Sørensen, Polarization transfer experiments in highresolution NMR spectroscopy, Prog. NMR Spectrosc. 21, 503569 (1989).

${ }^{19}$ N. Nielsen, O. Sørensen, Conditional bounds on polarization transfer, J. Magn. Reson. Ser. A 114, 24-31 (1995).

${ }^{20}$ N. C. Nielsen, T. Schulte-Herbrüggen, O. W. Sørensen, Bounds on spin dynamics tightened by permutation symmetry - application, Mol. Phys. 85, 1205-1216 (1995).

${ }^{21}$ J. Stoustrup, O. Schedletzky, S. J. Glaser, C. Griesinger, N. C. Nielsen, O. W. Sørensen, A Generalized Bound on Quantum Dynamics: Efficiency of Unitary Transformations between NonHermitian States, Phys. Rev. Lett. 74, 2921-2924 (1995).

${ }^{22}$ S. J. Glaser, T. Schulte-Herbrüggen, M. Sieveking, O. Schedletzky, N. C. Nielsen, O. W. Sørensen, C. Griesinger, Unitary control in quantum ensembles, maximizing signal intensity in coherent spectroscopy, Science 280, 421-424 (1998).

${ }^{23}$ T. S. Untidt, S. J. Glaser, C. Griesinger, N. C. Nielsen, Unitary bounds and controllability of quantum evolution in NMR spectroscopy, Mol. Phys. 96, 1739-1744 (1999). 
I evitt, Symmetry constraints on spin dynamics: Applicaion to hyperpolarized NMR, J. Magn. Reson. 262, 91-99 (2016). Publishifiga neja, F. Kramer, S. J. Glaser, Optimal experiments for maximi ing coherence transfer between coupled spins, J. Magn. Reson. 173, 116-124 (2005).

${ }^{26}$ T. O. Reiss, N. Khaneja, S. J. Glaser, Broadband Geodesic Pulses for Three Spin Systems: Time-Optimal Realization of Effective Trilinear Coupling Terms and Indirect SWAP Gates, J. Magn. Reson. 165, 95-101 (2003).

${ }^{27}$ N. Khaneja, S. J. Glaser, R. Brockett, Sub-Riemannian Geometry and Time Optimal Control of Three Spin Systems: Quantum Gates and Coherence Transfer, Phys. Rev. A 65, 032301 (2002).

${ }^{28} \mathrm{H}$. Yuan, N. Khaneja, Time optimal control of coupled qubits under nonstationary interactions, Phys. Rev. A 72, 040301(2005).

${ }^{29}$ N. Khaneja, T. Reiss, C. Kehlet, T. Schulte-Herbrüggen, S. J. Glaser, Optimal control of coupled spin dynamics: design of NMR pulse sequences by gradient ascent algorithms, J. Magn. Reson. 172 296-305 (2005).

${ }^{30}$ Z. Tošner, T. Vosegaard, C. T. Kehlet, N. Khaneja, S. J. Glaser, N. C. Nielsen, Optimal control in NMR spectroscopy: Numerical implementation in SIMPSON, J. Magn. Reson. 197, 120-134 (2009).

${ }^{31}$ S. Machnes, U. Sander, S. J. Glaser, P. de Fouquières, A. Gruslys, S. Schirmer, T. Schulte-Herbrüggen, Comparing, optimising and benchmarking quantum control algorithms in a unifying programming framework, Phys. Rev. A 84, 022305 (2011).

${ }^{32}$ P. de Fouquieres, S. G. Schirmer, S. J. Glaser, I. Kuprov, Second order gradient ascent pulse engineering, J. Magn. Reson. 212, 412-417 (2011).

${ }^{33} \mathrm{~S}$. J. Glaser, U. Boscain, T. Calarco, C. P. Koch, W. Köckenberger, R. Kosloff, I. Kuprov, B. Luy, S. Schirmer, T. Schulte-Herbrüggen, D. Sugny, K. Wilhelm, Training Schrödinger's cat: quantum optimal control, Eur. Phys. J. D. 69, 279 (2015).

${ }^{34}$ K. Kobzar, T. E. Skinner, N. Khaneja, S.J. Glaser, B. Luy, Exploring the limits of broadband excitation and inversion pulses, J. Magn. Reson. 170, 236-243 (2004).

${ }^{35}$ K. Kobzar, T. E. Skinner, N. Khaneja, S.J. Glaser, B. Luy, Exploring the limits of broadband excitation and inversion pulses II.: RF-power optimized pulses, J. Magn. Reson. 194, 58-66 (2008).

${ }^{36}$ K. Kobzar, S. Ehni, T. E. Skinner, S. J. Glaser, B. Luy, Exploring the Limits of Broadband 90 and 180 Universal Rotation Pulses, J. Magn. Reson. 225, 142-160 (2012)

${ }^{37}$ M. Nimbalkar, B. Luy, T. E. Skinner, J. L. Neyes, N. I. Gershenzon, K. Kobzar, W. Bermel, S. J. Glaser, The Fantastic Four: A Plug 'n' Play Set of Optimal Control Pulses for Enhancing NMR Spectroscopy, J. Magn. Reson. 228, 16-31 (2013).

${ }^{38}$ J. L. Neves, B. Heitmann, T. O. Reiss, H. H. R. Schor, N. Khaneja, S. J. Glaser, Exploring the limits of polarization transfer efficiency in homonuclear three spin systems, J. Magn. Reson. 181, 126-134 (2006)

${ }^{39}$ N. Pomplun, B. Heitmann, N. Khaneja, S. J. Glaser, Optimization of electron-nuclear polarization transfer, Appl. Magn. Reson. 34, 331-346 (2008).

${ }^{40}$ N. Pomplun, S. J. Glaser, Exploring the limits of electronnuclear polarization transfer efficiency in three spin systems, Phys. Chem. Chem. Phys. 12, 5791-5798 (2010).

${ }^{41}$ M. Nimbalkar, R. Zeier, J. L. Neves, S. B. Elavarasi, H. Yuan, N. Khaneja, K. Dorai, S. J. Glaser, Multiple-spin coherence transfer in linear Ising spin chains and beyond: Numerically optimized pulses and experiments, Phys. Rev. A 85, 012325 (2012).

${ }^{42} \mathrm{~S}$. Ehni, B. Luy, BEBEtr and BUBI: J-compensated concurrent shaped pulses for ${ }^{1} \mathrm{H}_{-}{ }^{13} \mathrm{C}$ experiments, J. Magn. Reson. 232, 7-17 (2013)

${ }^{43}$ T. Vosegaard, C. Kehlet, N. Khaneja, S. J. Glaser, N. C. Nielsen, Improved excitation schemes for multiple-quantum magic-angle spinning for quadrupolar nuclei designed using optimal control theory, J. Am. Chem. Soc. 127, 13768-13769 (2005).

${ }^{44}$ T. Schulte-Herbrüggen, A. Spörl, N. Khaneja, S. J. Glaser, Op- timal control-cased efficient synthesis of building blocks of quantum algorithms seen in perspective from network complexity towards time complexity, Phys. Rev. A 72, 042331/1-7 (2005).

${ }^{45}$ N. I. Gershenzon, K. Kobzar, B. Luy, S. J. Glaser, T. E. Skinner, Optimal control design of excitation pulses that accommodate relaxation, J. Magn. Reson. 188, 330-336 (2007).

${ }^{46}$ T. Schulte-Herbrüggen, A. Spörl, N. Khaneja, S. J. Glaser, Optimal control for generating quantum gates in open dissipative systems, Phys. Rev. B 44, 154013 (2011).

${ }^{47}$ T. E. Skinner, N. I. Gershenzon, M. Nimbalkar, S. J. Glaser, Optimal control design of band-selective excitation pulses that accommodate relaxation and RF inhomogeneity, J. Magn. Reson. 217, 53-60 (2012).

${ }^{48}$ S. S. Köcher, T. Heydenreich, S. J. Glaser, Visualization and analysis of modulated pulses in magnetic resonance by joint timefrequency representations, J. Magn. Reson. 249, 63 (2014).

${ }^{49}$ F. Kramer, M. V. Deshmukh, H. Kessler, S. J. Glaser, Residual dipolar coupling constants: An elementary derivation of key equations, Conc. Magn. Reson. 21A, 10-21 (2004).

${ }^{50}$ L. Braunschweiler, R. R. Ernst, Coherence transfer by isotropic mixing: Application to proton correlation spectroscopy, J. Magn. Reson. 53, 521-528 (1983).

${ }^{51}$ A. J. Shaka, C. J. Lee, A. Pines, Iterative schemes for bilinear operators; application to spin decoupling, J. Magn. Reson. 77, 274293 (1988).

52 S. J. Glaser and J. J. Quant, Homonuclear and heteronuclear Hartmann-Hahn transfer in isotropic liquids, in Advances in Magnetic and Optical Resonance Vol. 19, 59-252 (1996), Ed.: W. S. Warren (Academic Press).

${ }^{53}$ S. J. Glaser, Coupling Topology Dependence of PolarizationTransfer Efficiency in TOCSY and TACSY Experiments, J. Magn. Reson. Series A 104, 283-301 (1993).

${ }^{54}$ T. Untidt, T. Schulte-Herbrüggen, B. Luy, S. J. Glaser, C. Griesinger, O. W. Srensen, N. C. Nielsen, Design of NMR Pulse Experiments with Optimum Sensitivity. Coherence-OrderSelective Transfer in I2S and I3S Spin Systems, Molecular Physics, 95, 787-796 (1998).

${ }^{55}$ See Supplementary Material at [fill in doi here] for more details on the approaches of pulse sequence optimization, data of optimized pulse sequences, a product operator analysis of simple sequences consisting of two $90^{\circ}$ pulses separated by a delay, a more detailed analysis of time-optimal generation of double- and triple-quantum coherence in a system consisting of two and three coupled spins, details on determination of flip angles from spectrograms and information about the fidelity of frequency-selective spectrogram-based pulse sequences.

${ }^{56}$ R. Freeman, K. A. McLauchlan, J. I. Musher, K. G. R. Pachler, The relative signs of geminal and vicinal proton spin coupling constants, Mol. Phys. 5, 321 (1962).

${ }^{57}$ A. Garon, R. Zeier, S. J. Glaser, Visualizing operators of coupled spin systems, Phys. Rev. A 91, 042122 (2015).

${ }^{58}$ N. J. Glaser, M. Tesch, S. J. Glaser, 'SpinDrops', Version 1.2, itunes.apple.com (2015).

${ }^{59}$ V. Vijayan, J.-P. Demers, J. Biernat, E. Mandelkow, S. Becker, A. Lange, Low-Power Solid-State NMR Experiments for Resonance Assignment under Fast Magic-Angle Spinning, Chem. Phys. Chem., 10, 2205-2208 (2009).

${ }^{60}$ S. Hediger, B. H. Meier, N. D. Kurur, G. Bodenhausen, R. R. Ernst, NMR cross polarization by adiabatic passage through the Hartmann-Hahn condition (APHH), Chem. Phys. Lett. 223, 283288 (1994).

${ }^{61}$ G. A. Morris, R. Freeman, Selective excitation in Fourier transform nuclear magnetic resonance, J. Magn. Reson. 29, 433-462 (1978).

${ }^{62}$ K. Dorai, Arvind, A. Kumar, Implementing quantum-logic operations, pseudopure states, and the Deutsch-Jozsa algorithm using noncommuting selective pulses in NMR, Phys. Rev. A 61, 042306 (2000).

${ }^{63}$ N. Linden, B. Herv, R. J. Carbajoc, R. Freeman, Pulse sequences for NMR quantum computers: how to manipulate nuclear spins 
This manuscript was accepted by J. Chem.
while freezing the motion of coupled neighbours, Chem. Phys.
Lett. $305,28-34$ (1999).

Publish.i.jirgu nschweiler, R. R. Ernst, Coherence transfer by isotropic mixing. application to proton correlation spectroscopy, J. Magn. Reson. 53, 521-528 (1983).

${ }^{65}$ N. Khaneja, R. Brockett, S. J. Glaser, Time Optimal Control in Spin Systems, Phys. Rev. A 63, 032308 (2001).
${ }^{66}$ N. Khaneja, F. Kramer, S. J. Glaser, Optimal Experiments for Maximizing Coherence Transfer Between Coupled Spins, J. Magn. Reson. 173, 116-124 (2005).

${ }^{67}$ N. Chandrakumar, K. Nagayama, A New Experimental Method for Heternuclear Correlation Spectroscopy with CrossPolarization, Chem. Phys. Lett. 133, 288-292 (1987). 
$A$

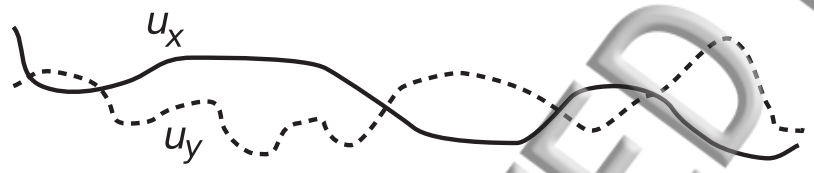

$T$

B
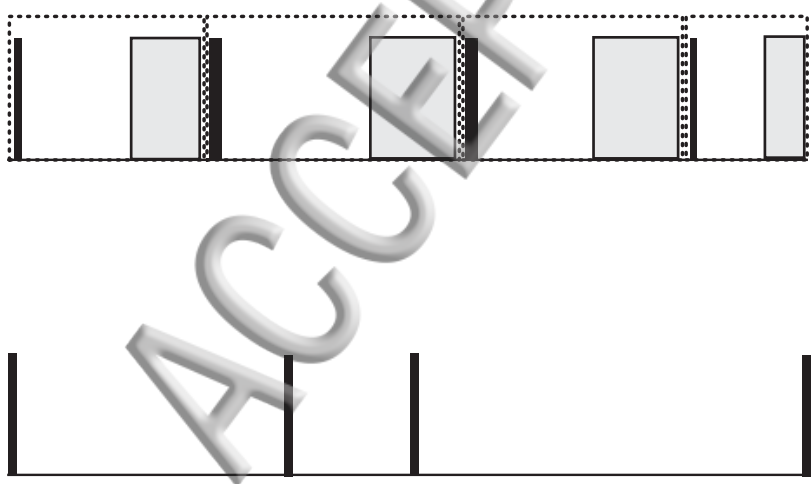

D 
$A$

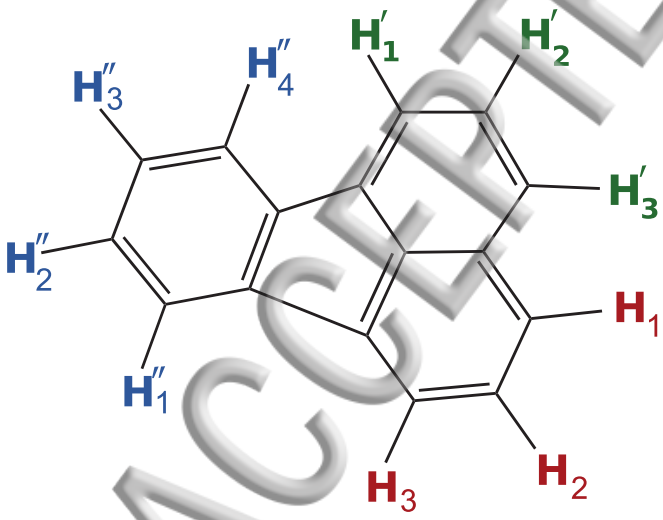

B

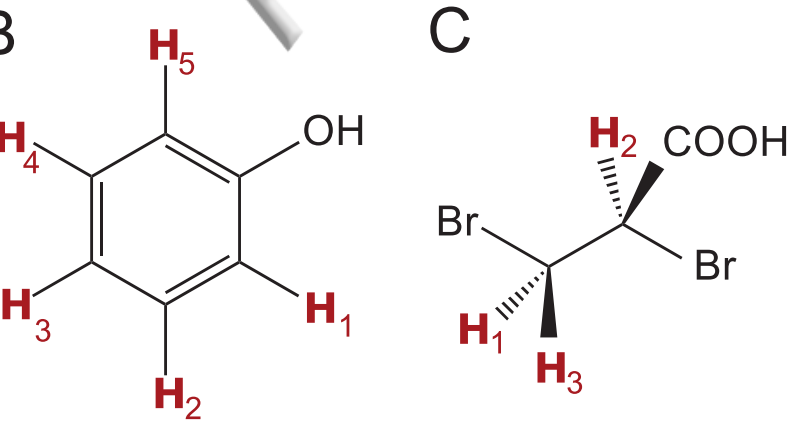




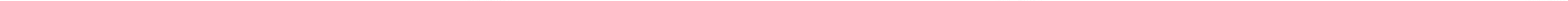




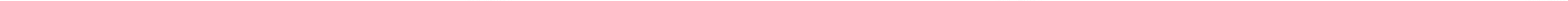




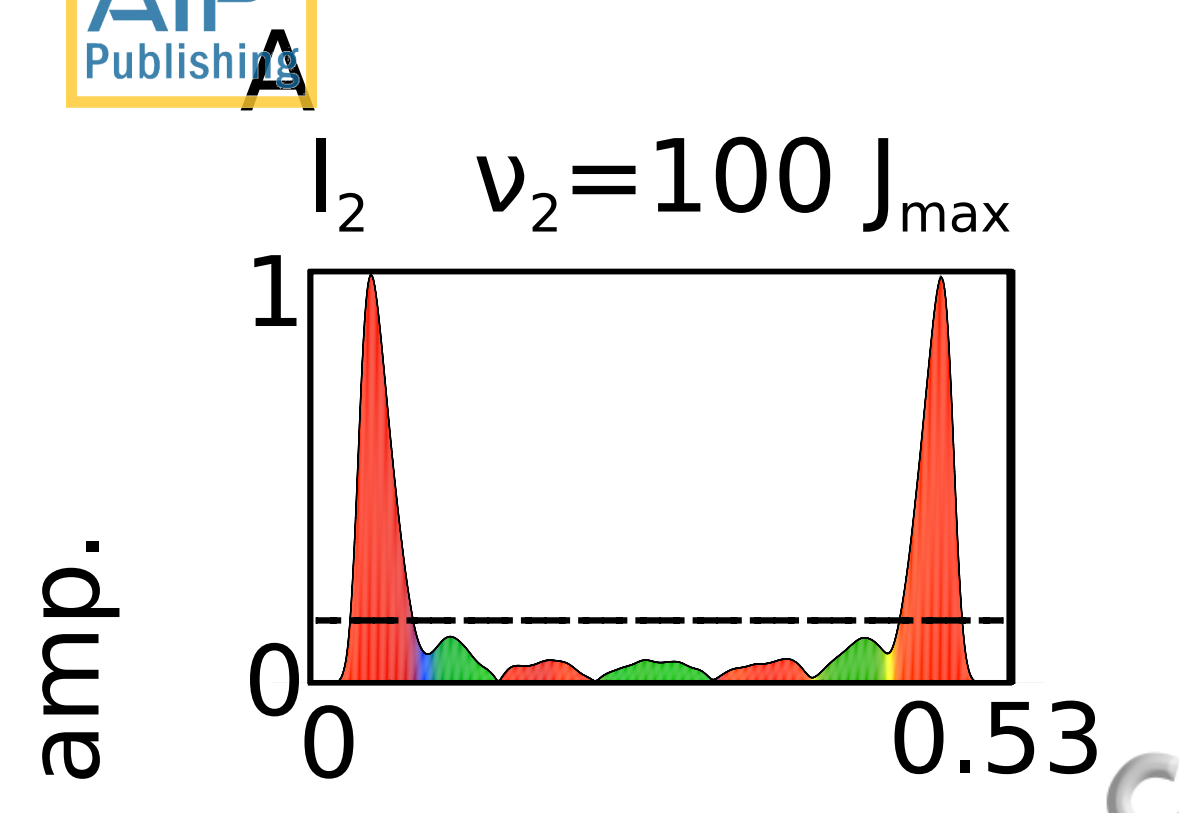

$A^{\prime}$

$(\pi / 2)_{\theta} \quad(\pi / 2)_{ \pm \theta}$

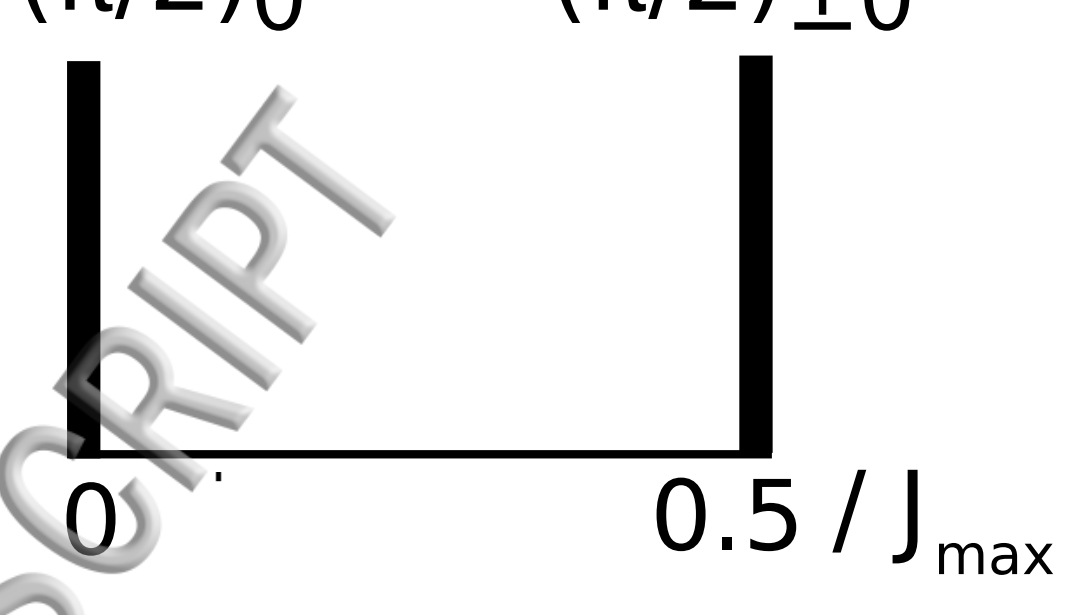

$\underbrace{I_{1} \quad v_{1}=0 J_{\max }}_{0}$

$t / J_{\max }^{-1}$

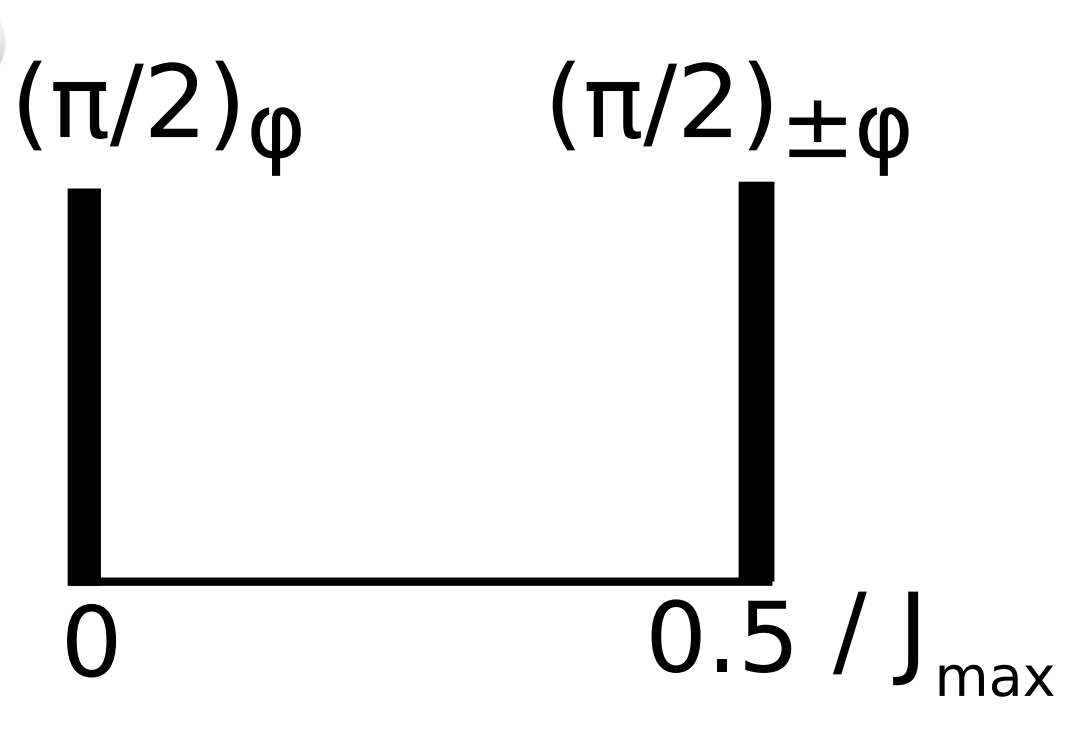

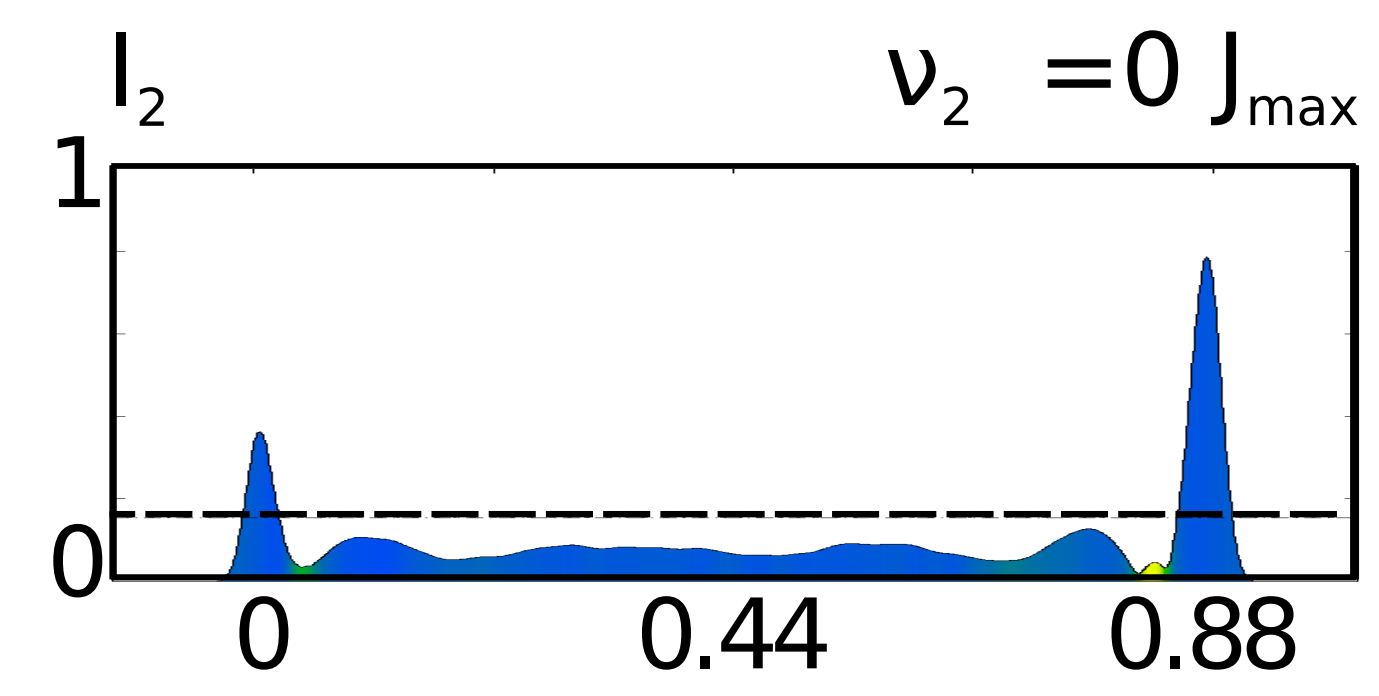

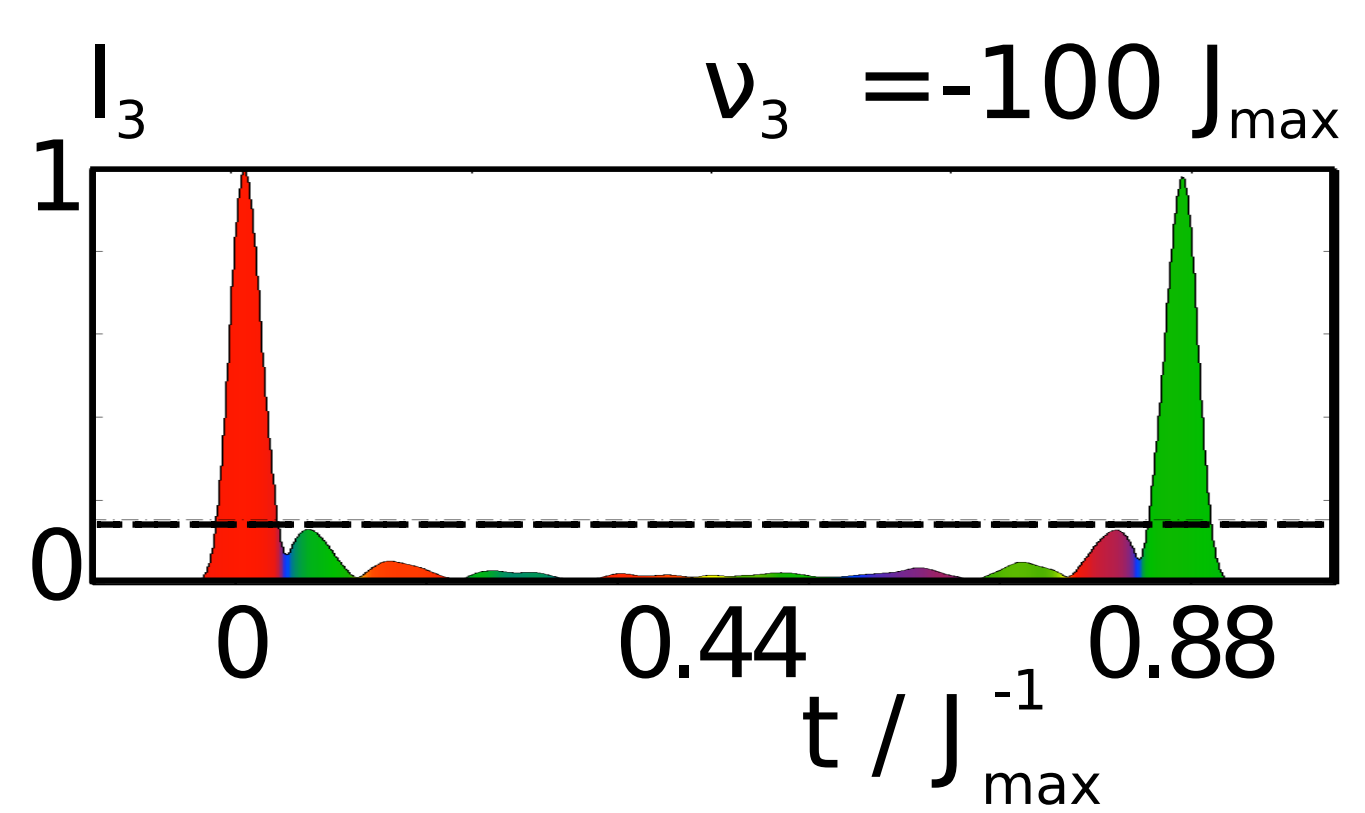

$(\pi / 2)_{\theta}$

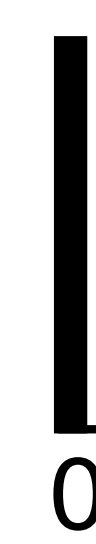

$(\pi / 2)_{ \pm \theta}$

$(\alpha)_{\varphi}$

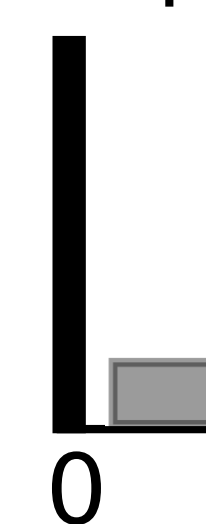

$(\pi / 2)_{\psi}$

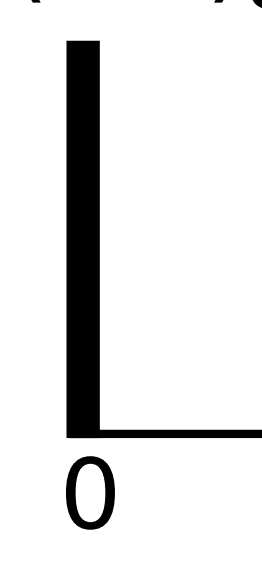

$(\pi)_{\varphi}$

$0.87 / \mathrm{J}_{\max }$

$( \pm \pi / 2-\alpha)_{\varphi}$

$.87 / J_{\max }$

$(\pi / 2)_{ \pm \psi}$

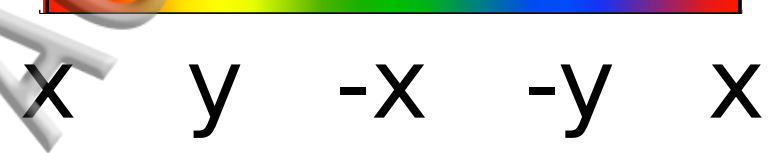


$90^{\circ}{ }_{-y}$

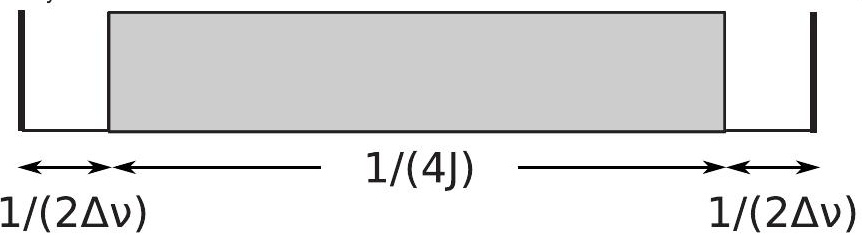



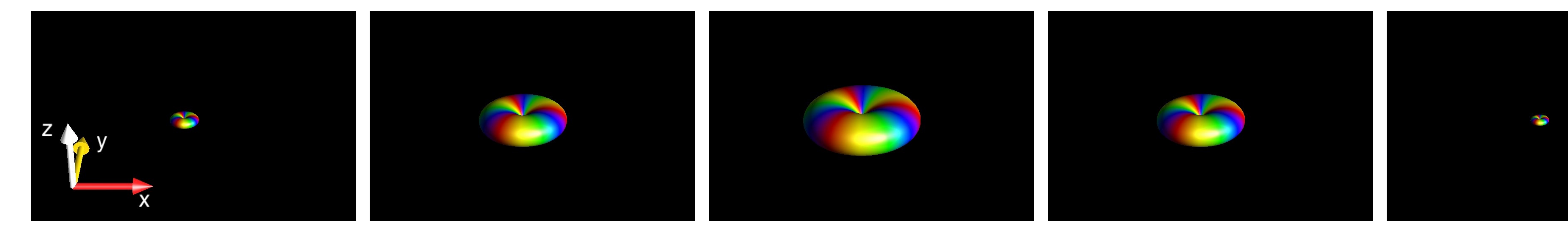

B

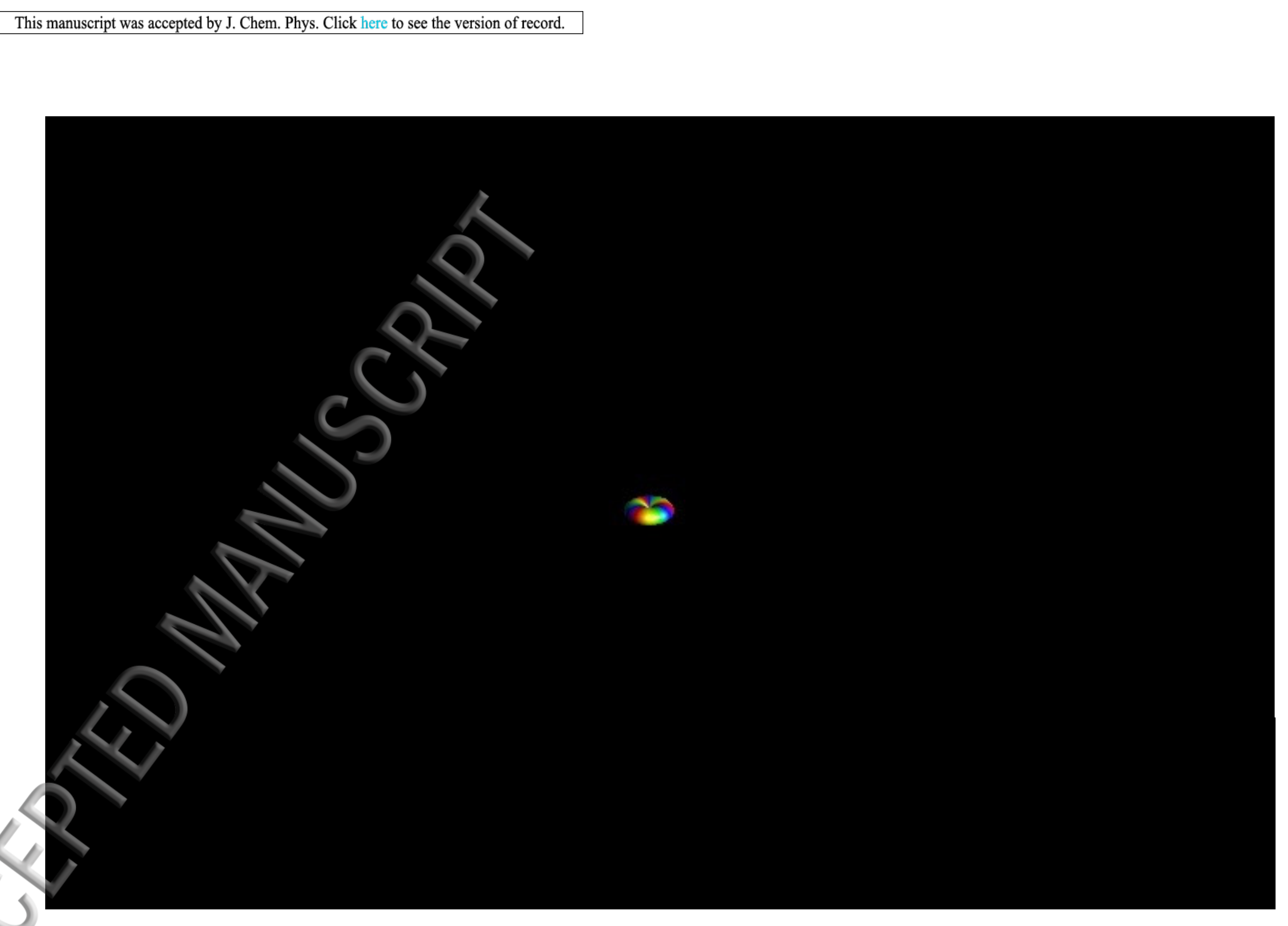

2

$\mathrm{T}=0.17 / \mathrm{J}^{-1} \max \quad \mathrm{T}=0.33 / \mathrm{J}^{-1} \max \quad \mathrm{T}=0.50 / \mathrm{J}^{-1} \max \quad \mathrm{T}=0.67 / \mathrm{J}^{-1} \max \quad \mathrm{T}=0.87 / \mathrm{J}^{-1} \max$ 


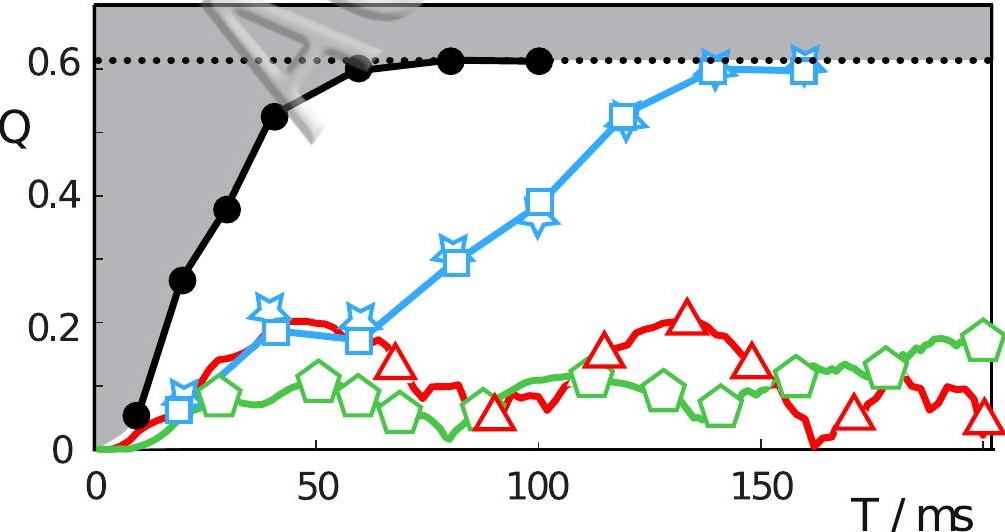

